\title{
Lupus-associated endogenous retroviral LTR polymorphism and epigenetic imprinting promote HRES-1/RAB4 expression and mTOR activation
}

\author{
Aparna Godavarthy, ${ }^{1}$ Ryan Kelly, ${ }^{1}$ John Jimah, ${ }^{1}$ Miguel Beckford, ${ }^{1}$ Tiffany Caza, ${ }^{1,2}$ David Fernandez, ${ }^{1,2}$ \\ Nick Huang, ${ }^{1,3}$ Manuel Duarte, ${ }^{1}$ Joshua Lewis, ${ }^{1}$ Hind J. Fadel, ${ }^{4}$ Eric M. Poeschla, ${ }^{4}$ Katalin Banki, ${ }^{5}$ \\ and Andras Perl ${ }^{1,2,3}$ \\ 'Division of Rheumatology, Department of Medicine, ${ }^{2}$ Department of Microbiology and Immunology, and ${ }^{3}$ Department \\ of Biochemistry and Molecular Biology, State University of New York, Upstate Medical University, College of Medicine, \\ Syracuse, New York, USA. ${ }^{4}$ Department of Molecular Medicine, Mayo Clinic College of Medicine, Rochester, New \\ York, USA. ${ }^{5}$ Department of Pathology, State University of New York, Upstate Medical University, College of Medicine, \\ Syracuse, New York, USA.
}

\begin{abstract}
Overexpression and long terminal repeat (LTR) polymorphism of the HRES-1/Rab4 human endogenous retrovirus locus have been associated with $\mathrm{T}$ cell activation and disease manifestations in systemic lupus erythematosus (SLE). Although genomic DNA methylation is diminished overall in SLE, its role in HRES-1/Rab4 expression is unknown. Therefore, we determined how lupusassociated polymorphic rs 451401 alleles of the LTR regulate transcription from the HRES-1/Rab4 promoter and thus affect T cell activation. The results showed that cytosine ${ }^{-119}$ is hypermethylated while cytosine ${ }^{-51}$ of the promoter and the LTR enhancer are hypomethylated in SLE. Pharmacologic or genetic inactivation of DNA methyltransferase 1 augmented the expression of HRES-1/Rab4. The minimal promoter was selectively recognized by metabolic stress sensor NRF1 when cytosine ${ }^{-119}$ but not cytosine ${ }^{-51}$ was methylated, and NRF1 stimulated HRES-1/Rab4 expression in human T cells. In turn, IRF2 and PSIP1 bound to the LTR enhancer and exerted control over HRES-1/Rab4 expression in rs451401 genotype- and methylation-dependent manners. The LTR enhancer conferred markedly greater expression of HRES-1/Rab4 in subjects with rs451401CC over rs451401CG alleles that in turn promoted mechanistic target of rapamycin (mTOR) activation upon T cell receptor stimulation. HRES-1/Rab4 alone robustly activated mTOR in human T cells. These findings identify HRES-1/ Rab4 as a methylation- and rs451401 allele-dependent transducer of environmental stress and controller of T cell activation.
\end{abstract}

Authorship note: $A G$ and RK contributed equally to this work.

Conflict of interest: The authors have declared that no conflict of interest exists.

Copyright: (c) 2020, American Society for Clinical Investigation.

Submitted: October 3, 2019 Accepted: November 26, 2019 Published: January 16, 2020.

Reference information: JCI Insight. 2020;5(1):e134010.

https://doi.org/10.1172/jci. insight.134010.

\section{Introduction}

Systemic lupus erythematosus (SLE) is a potentially fatal autoimmune disease of unknown etiology. Although the pathogenesis is incompletely understood, genetic and environmental factors are known be involved (1). Environmental factors, such as oxidative stress and infectious organisms, play crucial roles in modulating the expression of disease susceptibility genes through DNA methylation (2) and chromatin modification (3). Genomic DNA is overall undermethylated in patients with SLE, which preferentially affects genes that mediate interferon signaling $(4,5)$. Interferons are a major source of inflammation and disease manifestations in SLE (6, 7). Upon DNA demethylation, enhanced transcription of interferon-responsive genes involves activation of endogenous retroviral sequences (ERSs) (8). They make up as much as $8 \%$ of the human genome (9). They share regulatory elements with exogenous viruses and may serve as sensors of infections and mediators of autoreactivity (10, 11). The ERS HRES-1 is integrated at chromosome 1q42, a locus associated with lupus susceptibility (12). Polymorphic alleles of the retroviral long terminal repeat (LTR) are centered around a SNP, rs451401, which has been associated with autoimmunity and organ-specific manifestations in SLE $(13,14)$. This SNP has also been connected with susceptibility to multiple sclerosis (15-20). HRES-1 encodes a GTPase, HRES-1/Rab4, which is markedly overexpressed in T cells of SLE patients (21) and in lupus-prone mice before disease onset (22). 
Overexpression of HRES-1/Rab4 in lupus T cells reduces the expression of CD4 and CD3ל by promoting their endocytic recycling for lysosomal degradation $(21,23)$. Pharmacological inhibition of HRES-1/Rab4 blocks antinuclear antibody production and nephritis in lupus-prone mice (22). Therefore, we examined how HRES-1/ Rab4 expression is regulated and influenced by rs451401 polymorphism.

In this study, we reveal complex genetic and epigenetic mechanisms that regulate HRES-1/Rab4 expression. The minimal promoter harbors 2 potential NRF1-binding sites at nucleotides $-50 \rightarrow-67$ (site 1 ) and $-114 \rightarrow-124$ (site 2). NRF1 binds site 1 when cytosine ${ }^{-51}$ is unmethylated. It also binds site 2 when cytosine ${ }^{-119}$ is methylated. Interestingly, methylation of cytosine $e^{-51}$ was diminished 4.5 -fold while that of cytosine ${ }^{-119}$ was increased 10 -fold in SLE patients. These epigenetic changes favored promoter binding by NRF1 that itself stimulated HRES-1/Rab4 expression in primary human T cells. Moreover, HRES-1/ Rab4 expression was enhanced by the LTR and mediated by IRF2 and PSIP1 depending on rs451401 polymorphism and methylation of adjacent cytosine ${ }^{+954}$. HRES-1/Rab4 expression was increased in subjects carrying lupus-associated rs451401/CC LTR alleles that in turn promoted CD3/CD28-induced mechanistic target of rapamycin (mTOR) activation, which is hereby identified as a genetically defined checkpoint in the pathogenesis of SLE.

\section{Results}

HRES-1/Rab4 transcription is initiated from a 5' minimal promoter and enhanced by a polymorphic LTR within intron 1. The promoter of HRES-1/Rab4 transcription was mapped within a 545-bp upstream DNA (Figure 1A). Successive truncations localized the minimal promoter to a 127-bp DNA, which exhibited increased activity relative to larger fragments, $223 \mathrm{bp}\left(4.9\right.$-fold, $\left.P=2 \times 10^{-5}\right)$ and $545 \mathrm{bp}(4.3$-fold, $P=2.5$ $\left.\times 10^{-5}\right)$, respectively (Figure $\left.1 \mathrm{~B}\right)$. Bioinformatic analyses (24-26) identified 2 regions prominently targeted by transcription factors (TFs), nucleotides $-50 \rightarrow-67$ and $-114 \rightarrow-124$ (Supplemental Figure 1; supplemental material available online with this article; https://doi.org/10.1172/jci.insight.134010DS1). Given that both domains harbor NRF1 consensus sequences (GCGCA), they were designated as NRF1 sites 1 and 2, respectively (Figure 1A). Deletion of site 1 reduced promoter activity $(-67.1 \% ; P=1.5 \times$ $\left.10^{-8}\right)$, while that of site 2 increased promoter activity 2.2 -fold $\left(P=4.5 \times 10^{-9}\right.$; Figure $\left.1 C\right)$. Deletion of sites 1 and 2 obliterated promoter activity far beyond that of site 1 alone $\left(-79.8 \%, P=2.1 \times 10^{-9}\right.$; Figure 1C). This indicated potential cooperativity between the 2 sites.

The LTR harbors an SNP, rs451401, which was initially detected by HindIII polymorphism (27) and designated as genotype I, with guanine and genotype II with cytosine at position ${ }^{+959}$ (Figure 1A and ref. 27). Genotype II alleles showed increased frequencies in SLE both in case-control and family studies $(13,14)$. This HindIII site is centrally located within the LTR (Figure 1A) that is flanked by typical inverted repeats and harbors a canonical transactivation region for HIV-1-tat (ref. 23 and Supplemental Figure 3). The LTR enhanced transcription from the $5^{\prime}$ promoter (Figure 1D). Importantly, the lupus-linked genotype II (rs451401C) LTR conferred greater enhancer activity in HeLa cells, which was further increased in HeLa-tat cells (Figure 1D).

Methylation changes in HRES-1/Rab4 genomic locus are sequence specific - NRF1 site 2 is hypermethylated while NRF1 site 1 and the LTR enhancer are hypomethylated in SLE. The methylation state of 27 CpG motifs in the HRES-1/Rab4 promoter was examined in genomic DNA of 165 White female SLE patients and 109 matched White female controls by PCR amplification and bisulfite sequencing (Figure 2A). These CpGs were located between nucleotide positions -181 and -34 relative to the transcription start site. The average methylation level was increased at these 27 positions in SLE patients (controls: $2.3 \% \pm 0.10 \%$, SLE: $3.0 \%$ $\pm 0.18 \%, P=0.001)$. After Bonferroni correction, 15 of 27 sites showed significant changes in SLE $(P<$ 0.05 for 27 sites $=0.001852$ ). Methylation of 4 of 15 altered CpGs was decreased, 3 of which mapped to NRF1 site 1 (Figure 2A). In contrast, methylation was increased in NRF1 site 2 (Figure 2A). The CpG at 229406711, which is 170 bases upstream from the transcription start site, was also hypermethylated in SLE (controls: $1.9 \% \pm 0.30 \%$, SLE: $3.4 \% \pm 0.26 \%, P=7.4 \times 10^{-5}$ ) in accordance with earlier findings (Supplemental Figure 2 and ref. 4).

Given that rs451401 influenced enhancer activity of the LTR, we determined the methylation state of 3 flanking CpGs in 129 lupus patients and 111 matched healthy controls. Methylation of CpG 229407896 was reduced from $6.0 \% \pm 0.3 \%$ in controls to $3.6 \% \pm 0.2 \%$ in SLE patients $\left(P=3.4 \times 10^{-9} ;\right.$ Figure $\left.2 \mathrm{~B}\right)$. Likewise, methylation of CpG 229407737 was reduced, albeit to a lesser extent, from $12.6 \% \pm 0.4 \%$ in controls to 10.9 $\pm 0.3 \%$ in SLE $(P=0.001)$. Although methylation of CpG 229407834, which is 4 bases away from rs 451401 , 
A
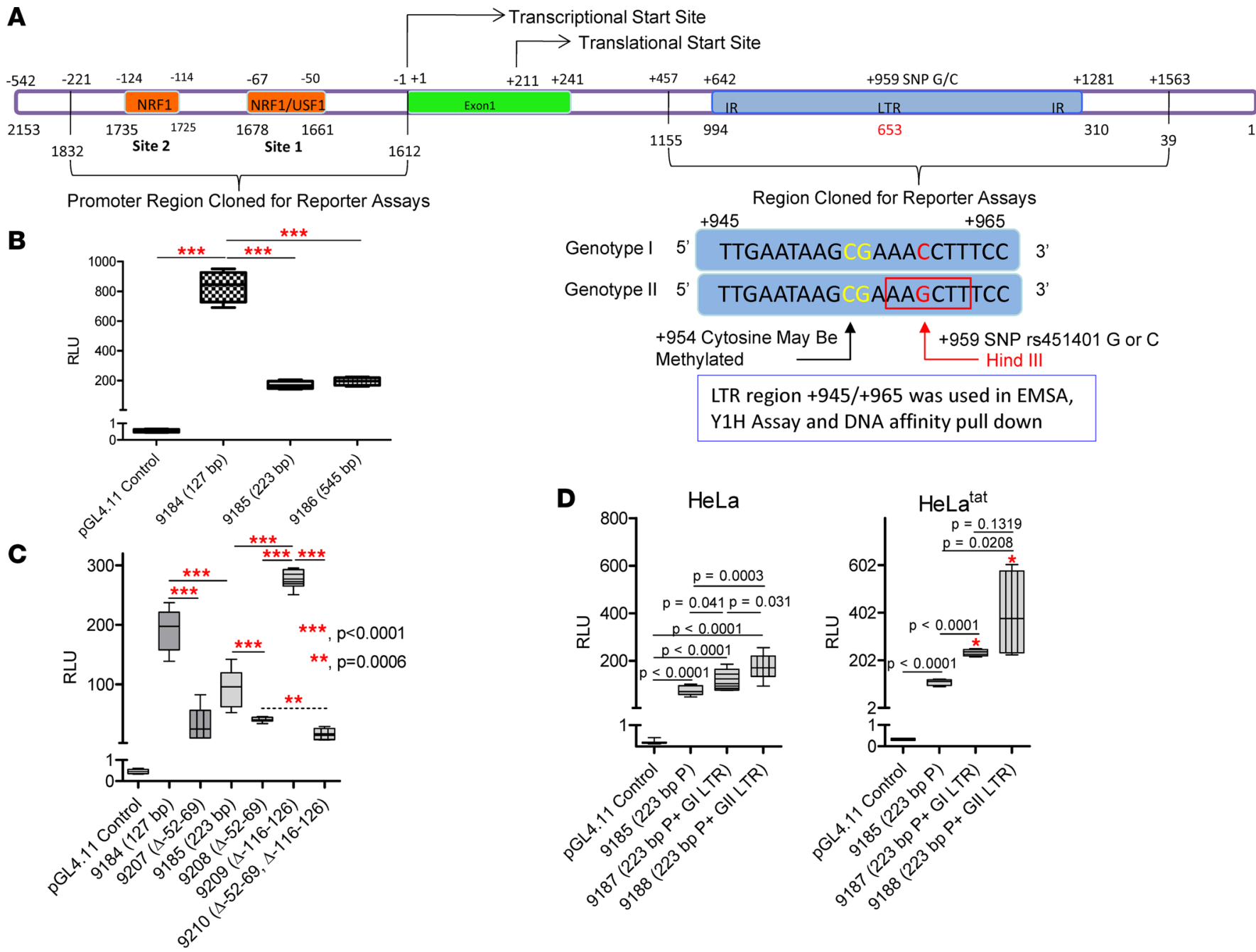

Figure 1. Delineation of transcriptional regulatory elements in the HRES-1/Rab4 genomic locus. The minimal promoter of HRES-1/Rab4 transcription was mapped to a 545-bp upstream DNA fragment using pGL4.11 firefly luciferase reporter plasmid that was cotransfected with pGL4.74 renilla luciferase control plasmid to equalize for transfection efficiency. (A) Schematic diagram of transcription factor-binding sites for NRF1 and NRF1/USF1 between nucleotides -50 to -67 and -114 to -124 , respectively, within the $5^{\prime}$ promoter. The SNP rs451401 is depicted with the LTR region of intron 1 . $G \rightarrow C$ transition of rs451401 creates a recognition site for Hind III restriction enzyme. (B) Localization of the minimal promoter to a 127-bp upstream DNA segment by deletion mapping within pCL4.11 expression vectors producing luciferase enzyme. (C) Effect of separate and combined deletions of NRF1 sites 1 and 2 on transcriptional activation by the minimal promoter. ${ }^{* *} P=0.0006$; ${ }^{* *} P<0.0001$. (D) Enhancement of promoter activity by the LTR region of intron 1 harboring the rs $451401 \mathrm{C}$ or rs $451401 \mathrm{C}$ allele. Data represent mean $\pm \mathrm{SEM}$ of 4 or more experiments. $P$ values represent comparison using 2 -tailed paired $t$ test, which reflect hypothesis testing and have not been corrected for multiple comparisons.

was not affected across all patients (Figure 2B), it was reduced in patients carrying 1 or $2 \mathrm{C}$ alleles relative to GG homozygote patients ( $P=0.003$; Figure 2C). Methylation at CpG positions 229407896 and 229407737 was diminished in SLE patients carrying $2 \mathrm{G}$ alleles (Figure 2C). As a control locus, CpGs similarly positioned in intron 1 of the IGF1 gene on chromosome 12 showed nearly identical methylation levels, reflecting an extremely tight control of this epigenetic process (chromosome 12 CpG 102796547: control, $87.8 \% \pm$ 2.6\%; SLE, 87.8\% \pm 0.6\%; CpG 102796637: control, $60.5 \% \pm 2.5 \%$; SLE: $61.9 \% \pm 0.9 \%$; Figure $2 \mathrm{~B}$ ).

DNMT1 regulates HRES-1/Rab4 expression. DNA hypomethylation has been implicated in drug-induced lupus (28). We found that procainamide stimulated HRES-1/Rab4 expression (Figure 3A). Hypomethylation has been attributed to inactivation of DNA methyltransferase 1 (DNMT1) in SLE (2). Interestingly, HRES-1/Rab4 expression was greater in cells lacking DNMT1 than controls with wild-type DNMT1 ( $P=0.006$, Figure 3B). HRES-1/Rab4 levels were slightly reduced upon deletion of DNMT3b, which may be attributed to upregulation of DNMT1 in these cells (Figure 3B). Increased expression of HRES-1/Rab4 was comparable between cells lacking DNMT1 only and those lacking both DNMT1 and DNMT3b (Figure 3B). Thus, HRES-1/Rab4 is directly regulated by DNMT1. 


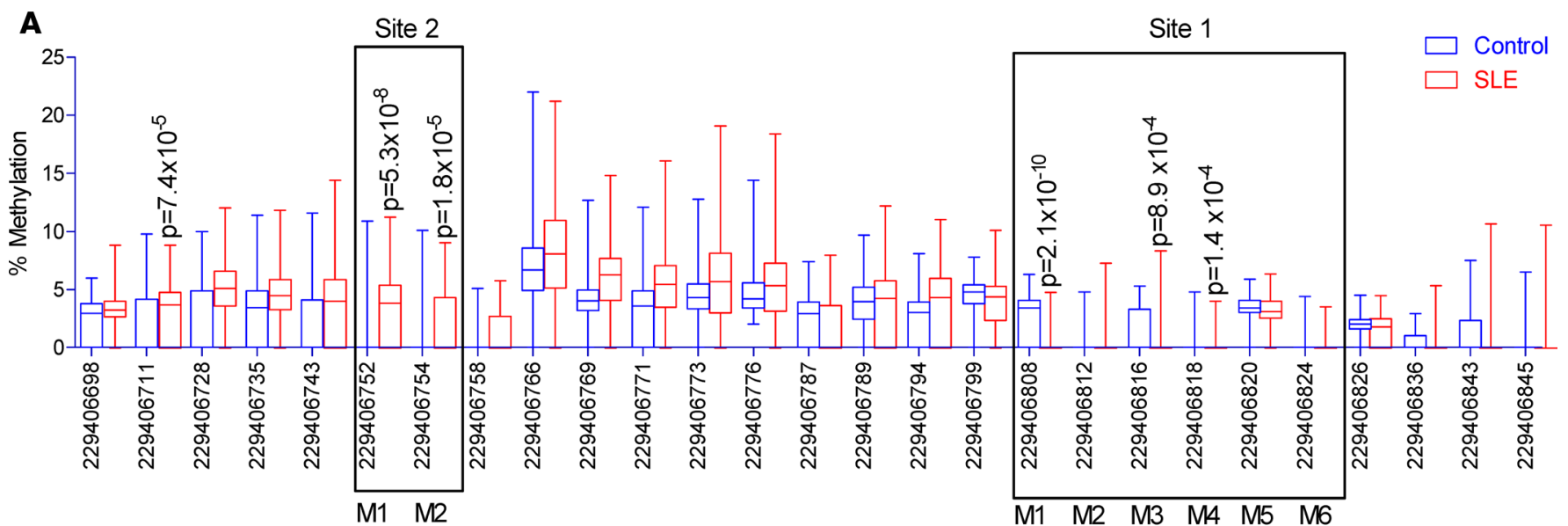

B
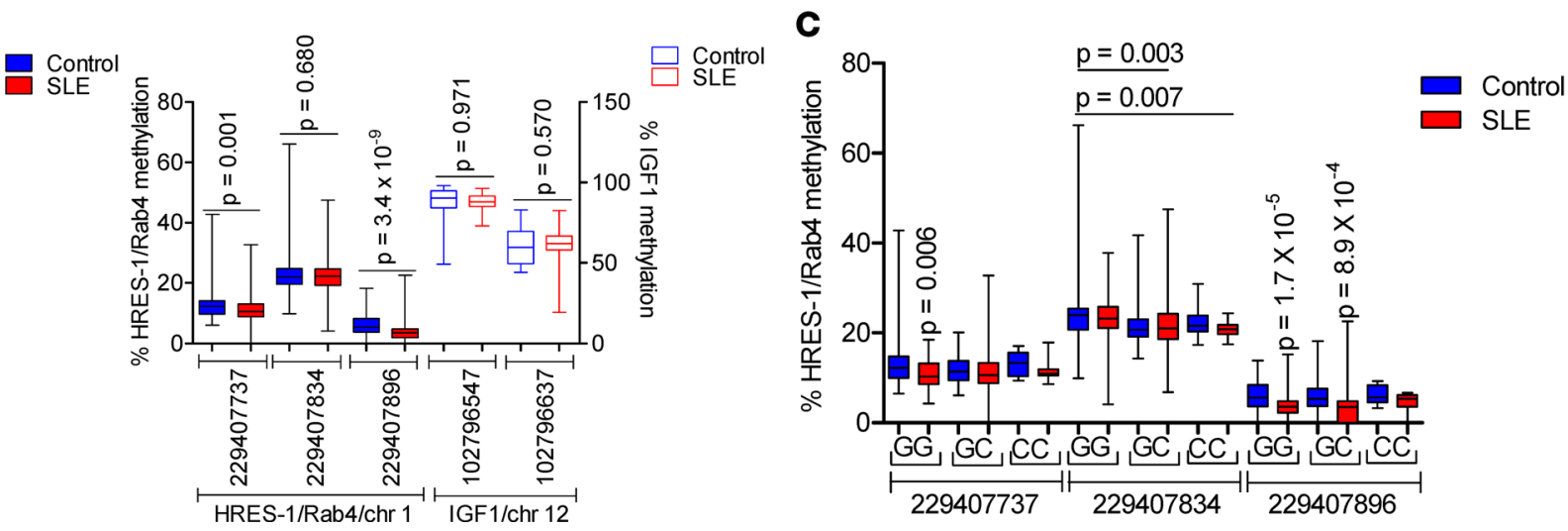

Figure 2. Methylation state of CpG motifs in the HRES-1/Rab4 promoter and LTR enhancer in genomic DNA from monocyte-depleted PBLs of 165 White female SLE patients and 111 healthy controls matched for sex (all female), ethnicity (all White), and age within a decade. (A) The methylation state of $27 \mathrm{CpC}$ motifs in the HRES-1/Rab4 promoter was examined by PCR amplification and bisulfite sequencing. These CpG residues were located between nucleotide positions -181 and -34 relative to the transcription start site, i.e., chromosome 1 base positions $229406661-229407517$ in build 37 of the human genome (GRCh37/19). (B) Methylation state was examined by bisulfite sequencing at 3 CpG motifs flanking SNP rs451401 in the LTR of intron 1 between chromosome 1 base positions 229407673 and 229407944 in build 37 of the human genome (CRCh37/19). As a control, methylation of CpC motifs in intron 1 of human insulin-like growth factor 1 (IGF1 or somatomedin C), which corresponds to base positions 102796547 and 102796637 of chromosome 12 , was analyzed. (C) Effect of rs451401 alleles on methylation state of CpG motifs in the HRES-1 LTR. Data are presented as box-and-whiskers plots. $P$ values represent comparison using 2-tailed unpaired $t$ test, which have been corrected for multiple comparisons.

NRF1 and USF1 bind the HRES-1/Rab4 promoter in methylation-dependent manner. Although the promoter lacks a TATA box, it contains 2 binding motifs for NRF1 (refs. 29, 30, and Figure 1A). Moreover, an E-box consensus sequence for USF1 (CACGTG) (31) is embedded within NRF1 site 2 (Figure 4A). In agreement with earlier studies showing induction of HRES-1/Rab4 protein expression by NO (21), NOC18 directly stimulated promoter activity (Supplemental Figure 5). Notably, NO induces NRF1 (32). Electrophoretic mobility shift assays (EMSAs) demonstrated TF binding to NRF1 sites within the promoter (Figure 4, B and C). Supershift analyses of unmethylated DNA revealed that NRF1 itself was responsible for binding site 1 (Figure 4B) but not site 2 (Figure 4C). In contrast, USF1 bound to site 2 (Figure 4C) but not site 1 (Figure 4B). Given that genomic DNA is partially methylated even in transcriptionally active promoters, we selectively methylated each CpG, M1-M6 in site 1 and M1 and M2 in site 2 (Figure 4A). NRF1 binding to site 1 was profoundly reduced by $\mathrm{CpG}$ methylation at M1 (cytosine ${ }^{-51}$, chromosome 1 base position 229406808) and, to a lesser extent, at M3 (cytosine ${ }^{-43}$, chromosome 1 base position 229406816; Supplemental Figure 6). Methylation did not affect USF1 binding to site 1 (Supplemental Figure 6). However, methylation influenced NRF1 binding to site 2 (Supplemental Figure 7). While methylation of the first $\mathrm{CpG}$ residue failed to have any effect, that of the second CpG (cytosine ${ }^{-119}$, chromosome 1 base position 229406758) eliminated USF1 binding and established NRF1 binding to site 2 (Supplemental Figure 7).

IRF1, IRF2, and PSIP1 bind the HRES-1 LTR depending on rs451401 genotype and DNA methylation. TF binding to the LTR was influenced by rs451401 (Supplemental Figure 8A). ${ }^{32} \mathrm{P}$-labeled LTR formed 7 complexes 
A
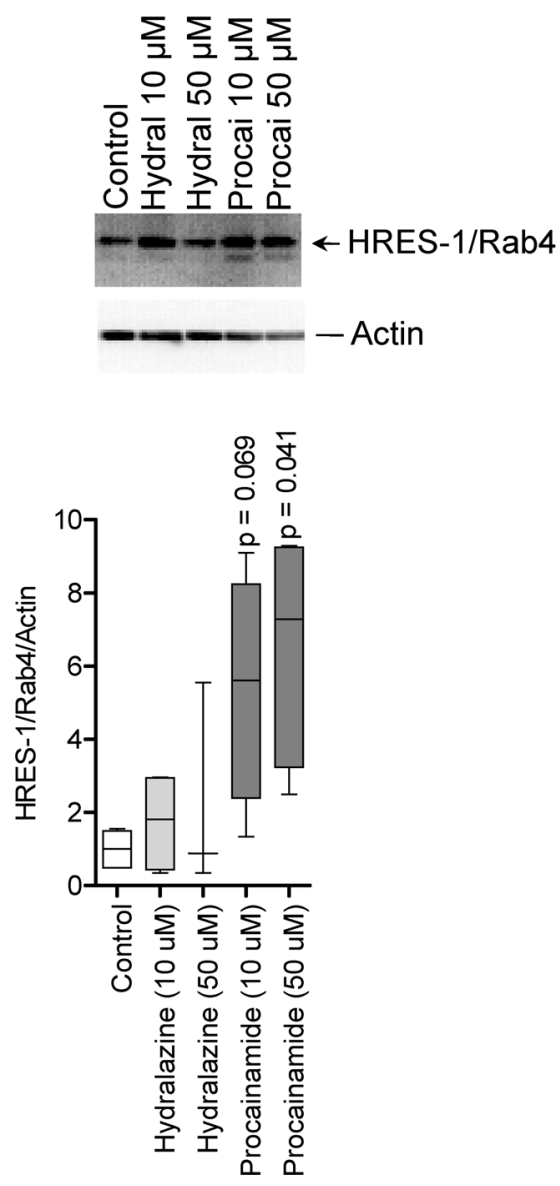

B
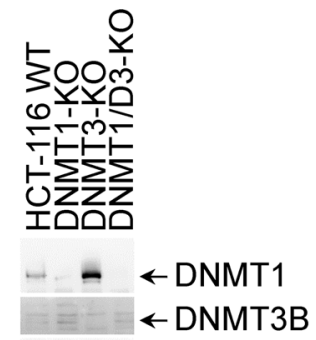

$-\sim-\leftarrow$ HRES-1/Rab4

---- Actin

$\leadsto-\infty$ HRES-1/Rab4

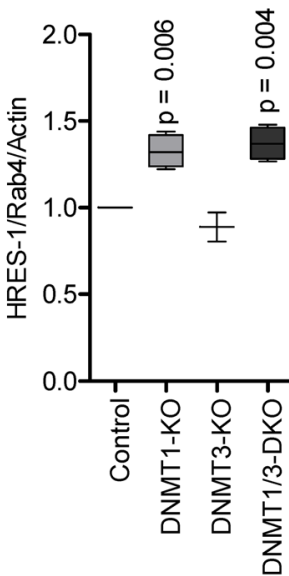

Figure 3. Expression of HRES-1/Rab4 is regulated by DNMT1. (A) Expression of Rab4A is stimulated by treatment of Jurkat cells with hydralazine and procainamide over 48 hours. Top: representative Western blots. Bottom: cumulative analyses. (B) Western blot analysis of Rab4A relative to $\beta$-actin in HCT-166 colon carcinoma cells lacking DNMT1 (line no. 30), DNMT3b (line no. 38), or both DNMT1 and DNMT3b (DNMT1/3-DKO, line no. 343) as well as in HCT-166 colon carcinoma cells with wild-type DNMT1 and DNMT3 alleles (line no. 28). Top: representative Western blots. Bottom: cumulative analyses. Data represent mean \pm SEM. $P$ values represent comparison using 2 -tailed paired $t$ test, which reflect hypothesis testing and have not been corrected for multiple comparisons.

with nuclear proteins from Jurkat human T cells (C1-C7; Supplemental Figure 8B). The formation of these complexes was abrogated by a 100-fold molar excess of cold oligonucleotides but was unaffected by a 100 fold excess of human transaldolase TAL-H_P12 promoter DNA (33), used as a nonspecific competitor (data not shown). Ten-fold or one hundred-fold molar excess of cold genotype II (rs451401/C) LTR blocked the formation of all complexes formed by either genotype (Supplemental Figure 8B). However, 100-fold excess of cold genotype I (rs451401/G) LTR did not block C6 formation by genotype II LTR (Supplemental Figure 8B). Thus, C6 was only formed with genotype II LTR.

The HRES-1 LTR harbors binding motifs for IRF1 and IRF2 within nucleotides 647-667 that include rs451401 (Supplemental Figure 8A). Supershift assays revealed that DNA-protein complexes C2 and C3 were formed by IRF1 and IRF2, respectively (Supplemental Figure 8C). IRF2 antibody also generated a shifted complex, C8 (Supplemental Figure 8C). As negative control, transaldolase antibody did not affect TF binding to the LTR (Supplemental Figure 8C). Of note, neither IRF1 nor IRF2 antibody affected C6 migration. Therefore, to uncover TFs responsible for genotype II allele-specific binding of the LTR, we performed yeast 1 -hybrid assay, using 3 copies of nucleotides $+945 \rightarrow+965$, harboring rs $451401 / \mathrm{C}$ in position $^{+959}$ as DNA bait (Figure 1). The cDNA library was made from Jurkat mRNA, and LTR-binding TFs were identified by DNA sequencing. TFs recognizing the LTR were independently assessed by sequencing of proteins isolated through binding of biotinylated oligonucleotides (Supplemental Figure 9). Moreover, we sequenced proteins in complex C6 excised from EMSA gels (Supplemental Figure 10). These independent approaches revealed that IRF1, IRF2, EWSR1, HSBP1, PARP1, PSIP1, SFRS3, and SFRS5 bind directly or indirectly to the LTR enhancer.

Since CpGs adjacent to rs451401 were hypomethylated in SLE (Figure 2C), we examined the influence of methylation on TF binding. Although none of the complexes binding to genotype I LTR were affected, the formation of C6 with genotype II LTR was abrogated by methylation (Supplemental Figure 11). A complex, designated as $\mathrm{CII}_{\mathrm{m}}$, was formed with methylated genotype II LTR (Supplemental Figure 11B). 
A
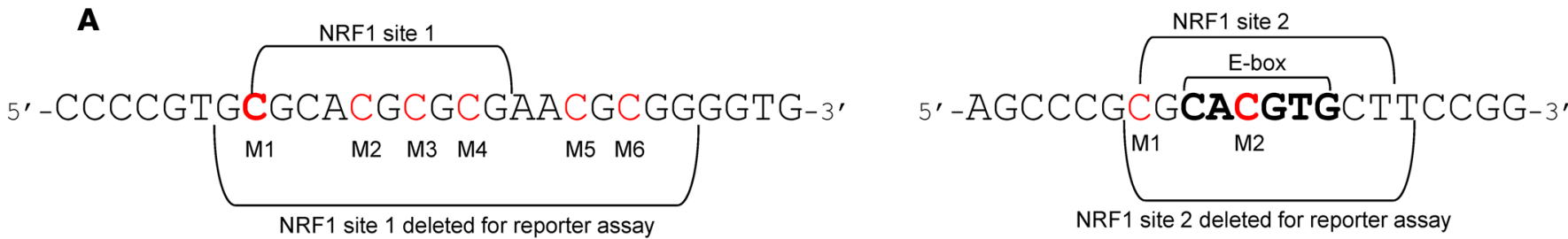

B

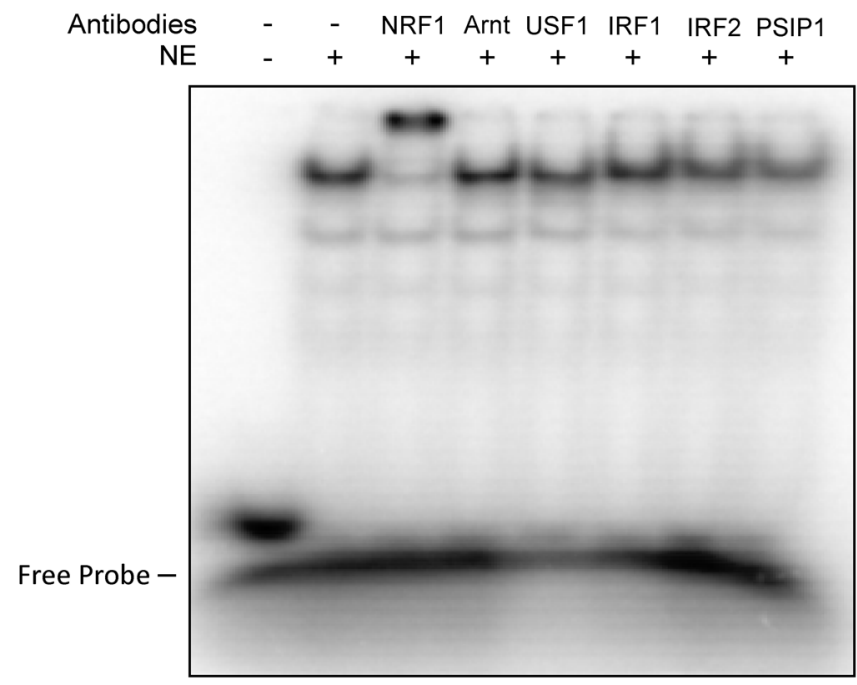

NRF1 site 1

\section{C}

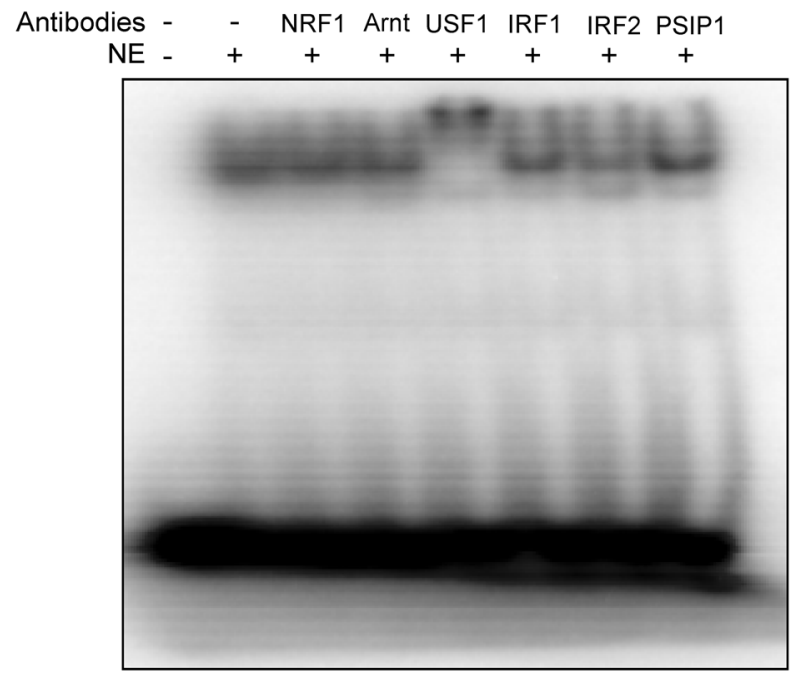

NRF1 site 2

Figure 4. NRF1 and USF1 bind to the minimal promoter of HRES-1/Rab4 in a methylation-dependent manner. (A) Sequences of oligonucleotides used to assess transcription factor (TF) binding to nucleotides -50 to -67 (nuclear respiratory factor 1-binding [NRF1-binding] site 1) and nucleotides -114 to -124 (NRF1-binding site 2) in the 5' HRES-1/Rab4 promoter. NRF1 site 2 also harbors an E-box consensus sequence for upstream stimulatory factor 1 (USF1) (CACGTC). Cytosine residues of $\mathrm{CpG}$ motifs are indicated in red. Cytosine ${ }^{-51}$ in NRF1 site 1 and cytosine-119 in NRF1 site 2, which profoundly affect TF binding upon methylation (Supplemental Figures 5 and 6), are bolded. (B) EMSA of TF binding in Jurkat nuclear extracts (NE) to NRF1 site 1 in the presence or absence of antibodies directed to NRF1, Arnt, USF1, IRF1, IRF2, and PSIP1. (C) EMSA of TF binding in Jurkat NE to NRF1 site 2 in the presence or absence of antibodies.

Supershift analyses detected no influence on TF binding to methylated genotype I LTR DNA (Supplemental Figure 11C). However, PSIP1 and IRF2 antibodies influenced complex formation with methylated genotype II LTR. PSIP1 antibody blocked $\mathrm{CII}_{\mathrm{m}}$ formation and elicited a complex with methylated genotype II LTR (Supplemental Figure 11D). IRF2 antibody reduced the formation of $\mathrm{CII}_{\mathrm{m}}$ (Supplemental Figure 11D). Methylation diminished the binding of IRF2 to genotype I LTR and increased its binding to genotype II LTR, as evidenced by formation of C8 (Supplemental Figure 12A).The ability of IRF2 to form C8 with unmethylated genotype II LTR was reduced by $54 \%$ relative to unmethylated genotype I LTR (Supplemental Figure 12B).

NRF1 and USF1 bind to the promoter and stimulate HRES-1/Rab4 expression in vivo. ChIP provided direct evidence for in vivo binding of NRF1 to sites 1 and 2 in Jurkat cells (Supplemental Figure 13A) and HeLa cells (Supplemental Figure 13B). USF1 showed binding to site 2 in all cell types (Supplemental Figure 13). As a positive control, NRF1 binding to the promoter of proton-coupled folate transporter was confirmed in vivo (Supplemental Figure 14). Both NRF1 and USF1 markedly increased HRES-1/Rab4 protein levels relative to $\beta$-actin in primary human $\mathrm{T}$ cells (Figure $5 \mathrm{~A}$ ).

IRF1, IRF2, PSIP1, HSBP1, SFRS3, and PARP1 bind to the LTR and regulate HRES-1/Rab4 expression. ChIP demonstrated binding of IRF1, IRF2, PSIP1, HSBP1, and SFRS3 to the LTR in vivo (Supplemental Figure 13C). HRES-1/Rab4 was downregulated by IRF1 in T cells of subjects with rs451401/ GG but not with rs451401/CC alleles (Figure 5B). IRF1 also suppressed HRES1/Rab4 expression in HeLa cells, which was relieved in HeLa-tat cells (Supplemental Figure 15). The suppression of HRES1/Rab4 by IRF-1 was corroborated in MCF7 cells with genotype I LTR (Supplemental Figure 4B) that overexpress IRF1 (Supplemental Figure 16). Along this line, dominant-negative IRF1 increased HRES-1/Rab4 expression (Supplemental Figure 16). 


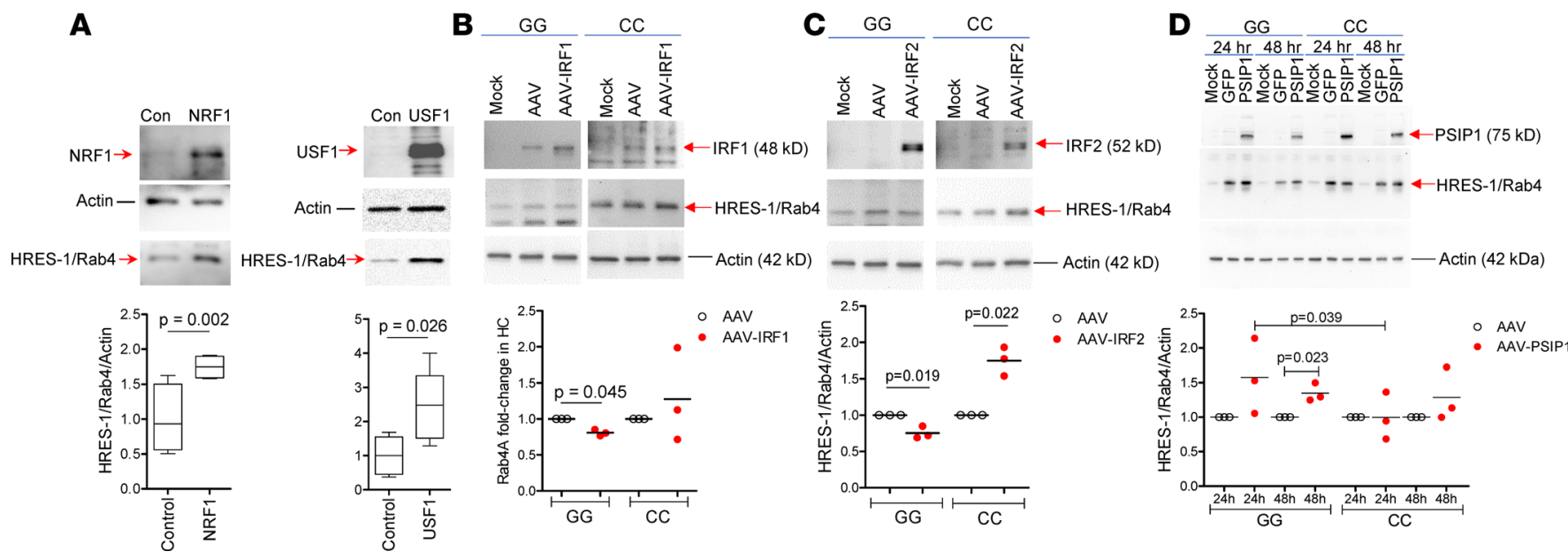

Figure 5. Regulation of HRES-1/Rab4 expression by TFs in primary human T cells. (A) Transduction of NRF1 and USF1 increases HRES-1/Rab4 protein levels relative to $\beta$-actin in normal human peripheral blood T cells. NRF1 and USF1 were transduced using pAAV-IRES-GFP expression vectors. Top: representative Western blots. Bottom: cumulative analysis of 4 or more independent experiments. $P$ values reflect comparison of cells transduced with NRF1 or USF1 relative to control cells transduced with AAV vector alone (Con) using paired $t$ test. (B-D) LTR allele-dependent regulation of HRES-1/Rab4 expression by IRF1 (B), IRF2 (C), and PSIP1 (D). Top: representative Western blots. Bottom: cumulative assessment of HRES-1/Rab4 protein levels relative to $\beta$-actin in 3 human subjects with rs451401CG (GC) alleles and 3 human subjects with rs451401CC (CC) alleles. $P$ values represent comparison using 2 -tailed paired $t$ test, which reflect hypothesis testing and have not been corrected for multiple comparisons.

Similar to IRF1, IRF2 reduced HRES-1/Rab4 expression in T cells of subjects with rs451401/GG alleles (Figure 5C). In contrast, IRF2 enhanced the expression of HRES-1/Rab4 in genotype II (rs451401/CC) subjects (Figure 5C). IRF2 also stimulated HRES-1/Rab4 expression in HeLa cells (Supplemental Figure 18A). Unlike the suppression by IRF1, the upregulation of HRES-1/Rab4 by IRF2 was not influenced by HIV-1-tat (Supplemental Figure 17A). IRF2 also reduced the expression of HRES-1/Rab4 in Jurkat cells with rs451401/GC alleles (Supplemental Figure 4C), which was reversed by HIV-1-tat (Supplemental Figure 17B).

PSIP1 stimulated HRES-1/Rab4 expression in subjects with genotype I (rs451401GG) LTR (Figure 5D). A permissive role for this TF in HRES-1/Rab4expression has been corroborated in cells lacking PSIP1 (Supplemental Figure 18).

SFRS3 increased HRES-1/Rab4 expression in peripheral blood lymphocytes (PBLs) (Supplemental Figure 19). Due to inefficient adeno-associated virus (AAV) transduction, the effect of SFRS5 could not be assessed in PBLs (data not shown). However, SFRS5 significantly upregulated HRES-1/Rab4 in HeLa and HeLa-tat cells (Supplemental Figure 20). HSBP1 reduced HRES-1/Rab4 expression in HeLa cells but not in HeLa-tat cells (Supplemental Figure 21).

HRES-1/Rab4 expression in human PBLs is influenced by rs 451401 in the LTR. To determine whether rs 451401 influences gene expression, HRES-1/Rab4 protein levels were compared between healthy White females carrying rs451401/GG or rs451401/CC alleles of the LTR with and without CD3/CD28 costimulation. While HRES-1/Rab4 protein levels were slightly increased at baseline in donors with rs451401/CC alleles, they were markedly elevated by CD3/CD28 costimulation in subjects with rs451401/GG alleles (14.4 $\pm 0.9-$ fold; $P=0.009)$ and subjects with rs $451401 / \mathrm{CC}$ alleles $(33.2 \pm 4.8$-fold; $P=0.011)$. Following CD3/CD28 costimulation, HRES-1/Rab4 protein levels showed a $2.3 \pm 0.3$-fold greater increase in donors with rs451401/CC alleles relative to donors with rs451401/GG alleles $(P=0.018$; Figure 6A). HRES-1/Rab4 protein levels were elevated 1.9-fold in SLE patients with rs451401/CC LTR relative to those with rs451401/GG LTR ( $P$ $=0.022$; Figure 6B). HRES-1/Rab4 expression was also increased in rs451401/CC patients $(2.3 \pm 0.3$-fold $)$ relative to rs451401/GG patients upon $\mathrm{CD} 3 / \mathrm{CD} 28$ costimulation $(1.6 \pm 0.1$-fold; $P=0.038$; Figure 6B). With respect to healthy controls, the extent of CD3/CD28-induced overexpression of HRES-1/Rab4 was constrained in patients with SLE with rs451401/GG $(P=0.0408)$ or rs $451401 / C C$ alleles $\left(P=3.4 \times 10^{-9}\right)$.

Since TCR activation markedly overstimulated the expression of HRES-1/Rab4 in subjects with rs451401/CC alleles, we examined the involvement of TFs binding to the promoter or LTR DNA (Figure 7A). CD3/CD28 costimulation induced the expression of USF1 and PSIP1 at greater degrees in subjects with rs451401/CC alleles relative to subjects with rs451401/GG alleles (Figure 7B). While IRF1 expression was not influenced by LTR genotypes, its molecular weight was altered due to 
A
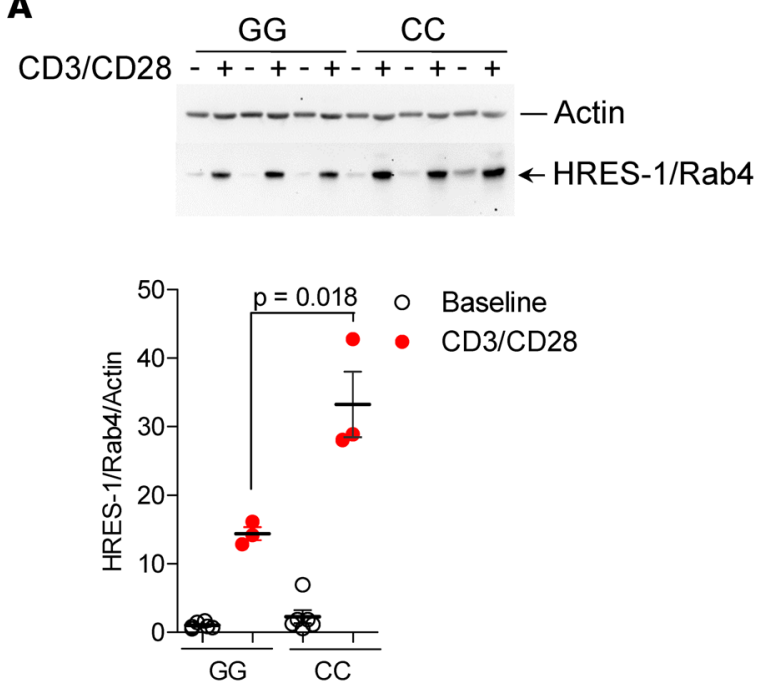

B

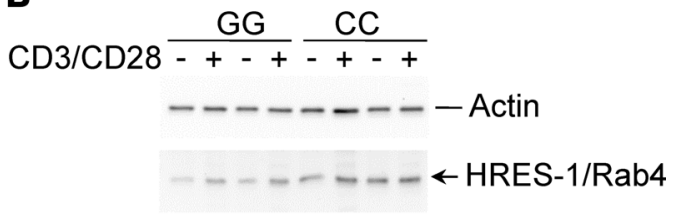

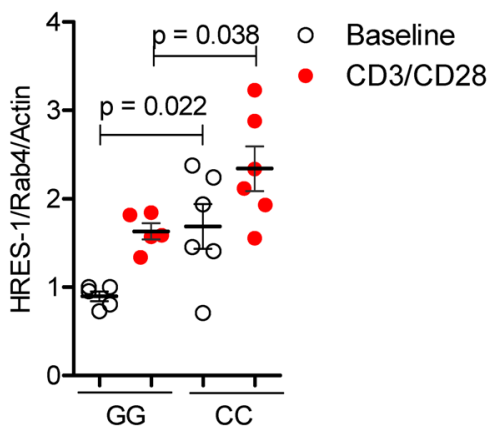

Figure 6. HRES-1/Rab4 expression is increased in PBLs that carry rs451401 CC alleles in the LTR enhancer. Western blot analyses were performed with and without CD3/CD28 costimulation for 72 hours. (A) Effect of T cell receptor stimulation on expression of HRES-1/Rab4 in healthy control subjects with homozygous GG or CC alleles of the HRES-1/Rab4 LTR. Top: Western blots. Bottom: cumulative analysis of 3 subjects with GC and 3 subjects with CC alleles. (B) Effect of T cell receptor stimulation on expression of HRES-1/Rab4 in 5 SLE patients with homozygous CG alleles and 6 patients with homozygous CC alleles of the HRES-1/Rab4 LTR. $P$ values represent comparison using 2-tailed paired $t$ test, which reflect hypothesis testing and have not been corrected for multiple comparisons.

anticipated posttranslational modification following T cell activation (34). Since mTOR localizes to HRES-1/Rab4-positive endosomes (21), we examined the effect of LTR polymorphism on TCR-induced mTOR activation. Protein levels of mTOR substrate 4E-BP1 were elevated in subjects with rs451401/CC alleles following CD3/CD28 costimulation (Figure 7C). We also observed a moderate increase of S6K protein levels in subjects with rs451401/CC alleles without TCR stimulation. Protein levels of HRES-1/Rab4 correlated with those of 4E-BP1, USF1, and PSIP1 (Figure 7D and Table 1). These findings imply that HRES-1/Rab4 overexpression in subjects with rs451401/CC alleles may exert a positive feedback onto TCR-mediated signal transduction.

HRES-Rab4 activates $m$ TORC1 and inhibits $m$ TORC2. Given that MTOR activation was enhanced in human subjects with the rs451401CC LTR genotype, we examined whether this change can be directly attributed to higher levels of HREs1/Rab4 expression. As shown in Figure 8, phosphorylation of mTORC1 substrates S6K and 4E-BP1 was increased by overexpression of HRES-1/Rab4, indicating activation of mTORC1. An opposite trend was observed upon overexpression of dominant-negative HRES-1/Rab4 ${ }^{\mathrm{S27N}}$ (HRES-1/Rab4-DN; Figure 8). Alternatively, phosphorylation of Akt was increased in cells overexpressing HRES-1/Rab4-DN, which is suggestive of greater mTORC2 activation (Figure 8). Moreover, regulation of mTOR activation by HRES-1/Rab4 was independently assessed in primary human T cells. Knockdown of HRES-1/Rab4 by siRNA reduced mTORC1 and increased mTORC2 activities, as evidenced by diminished pS6K and elevated pAkt levels, respectively (Figure 9).

\section{Discussion}

The present results delineate the genetic and epigenetic mechanism that controls HRES-1/Rab4 expression and reveal that mTOR pathway activation in human $\mathrm{T}$ cells is determined by polymorphism of the endogenous retroviral LTR. Since HRES-1/Rab4 and mTOR are expressed ubiquitously, this regulatory axis has broad implications for developmental and pathological pathways in cancer, inflammation, metabolic diseases, and aging, which are controlled through endosomal traffic and mTOR activation (35).

The HRES-1/Rab4 promoter and LTR enhancer interact with remarkably different TFs. The promoter is activated by NRF1 $(29,30)$ and USF1 $(31)$, which are capable of sensing oxidative (32) and metabolic stress (36), respectively. By contrast, the LTR is activated by HIV-1-tat and immune response-related TFs, notably IRF1, IRF2, PSIP1, SFRS3, SFRS5, and HSBP1 (Figure 10). 
A
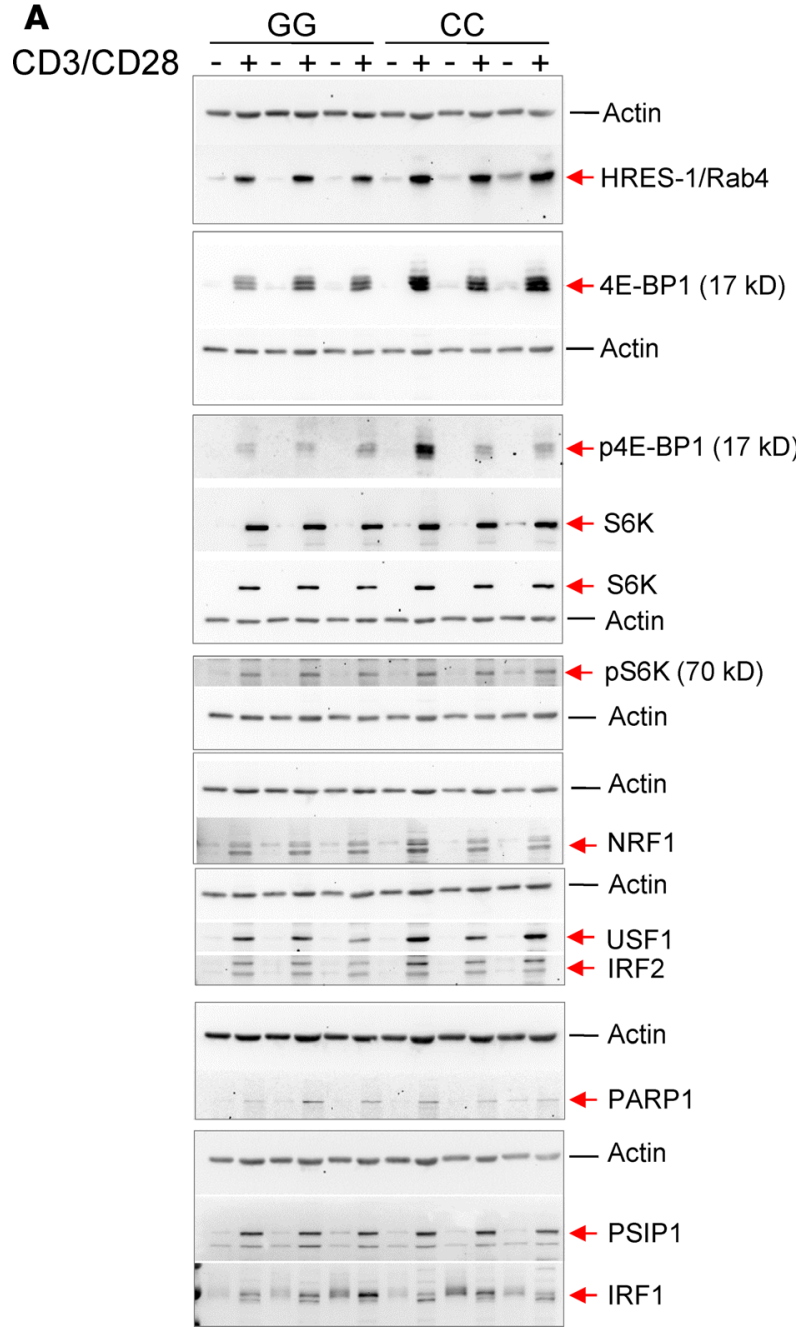

B

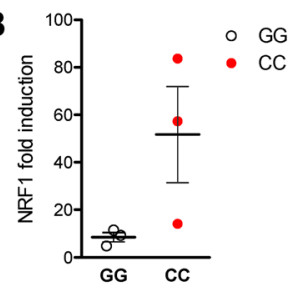

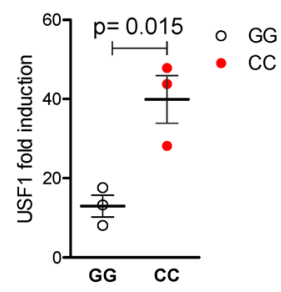

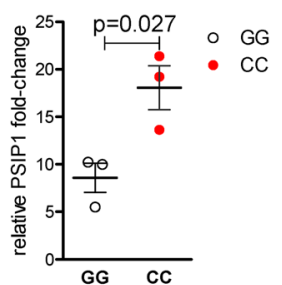

C

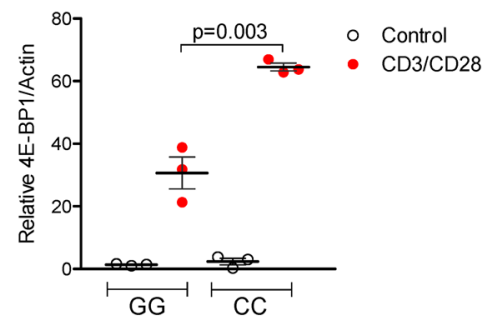

D

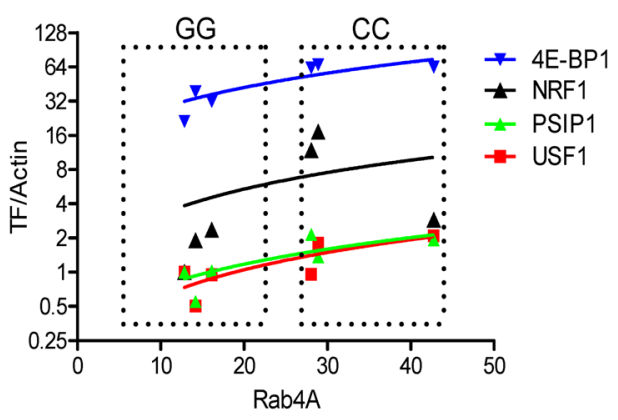

Figure 7. TCR stimulation-induced mTOR activation and TF expression are influence by rs451401 alleles of the HRES-1 LTR. (A) Effect of T cell receptor stimulation on mTOR activation, as measures by protein levels of key substrates, 4E-BP1 and S6K, and expression of transcription factors, NRF1, USF1, PARP1, PSIP1, IRF1, and IRF2. Western blot analysis was performed in 3 subjects with GG and 3 subjects with CC alleles, who have also been included as healthy controls in Figure 6A. (B) Cumulative analysis of 4E-BP1 protein levels. $P$ values reflect comparison with unpaired $t$ test. (C) Cumulative analysis of fold induction of TF protein levels in subjects with GC and CC alleles. (D) Linear regression analysis of 4E-BP1 and TF protein levels relative to HRES1/Rab4. Cumulative analysis of fold induction of TF protein levels in subjects with GG and CC alleles.

Binding of NRF1 and USF1 to the promoter was clearly methylation dependent. NRF1 binding to nucleotides $-50 \rightarrow-67$ was sensitive to cytosine ${ }^{-51}$ methylation. In contrast, USF1 solely bound nucleotides $-114 \rightarrow-124$ when cytosine $e^{-119}$ was methylated. Strikingly, cytosine $e^{-51}$ methylation was 4.5 -fold diminished while cytosine ${ }^{-119}$ methylation was 10 -fold increased in patients with SLE, favoring promoter binding by both NRF1 and USF1, which stimulated HRES-1/Rab4 expression in primary T cells. The minimal promoter was selectively recognized by metabolic stress sensor NRF1 when cytosine ${ }^{-119}$ but not cytosine $^{-51}$ was methylated, and NRF1 stimulated HRES-1/Rab4 expression in human T cells. While NRF1-binding site 1 (nucleotides $-50 \rightarrow-67$ ) stimulated promoter function, binding site 2 (nucleotides $-114 \rightarrow-124)$ acted as a repressor. The results also show that cytosine ${ }^{-119}$ was hypermethylated while cytosine $^{-51}$ of the HRES-1/Rab4 promoter was hypomethylated in SLE. Thus, SLE-associated epigenetic changes may favor HRES-1/Rab4 expression by facilitating NRF1 binding to both sites within the promoter and enabling the stimulatory function of a hypomethylated site 1 and restraining the repressor function of a hypermethylated site 2. Lupus-specific methylation changes occur in a cell type-specific manner, as suggested by the expansion of FoxP $3^{+}$Tregs, restraint of proinflammatory $\mathrm{CD} 3^{+} \mathrm{CD} 4{ }^{-} \mathrm{CD} 8^{-} \mathrm{T}$ cells, and clinical improvement in 5-azacytidine-treated MRL/lpr mice (37). 
Table 1. Pearson $r$ values and $P$ values of correlation analyses of 4E-BP1 and TF protein levels with HRES-1/Rab4.

\begin{tabular}{ccccc}
\hline $\mathbf{X}=$ HRES-1/Rab4 & 4E-BP1 & NRF1 & USF1 & PSIP1 \\
Pearson $r$ & 0.8545 & 0.3727 & 0.8536 & 0.8081 \\
$P$ value (2 tailed) & 0.0302 & 0.4669 & 0.0306 & 0.0517 \\
X refers to HRES-1/Rab4 protein levels. All protein levels were normalized to actin loading control. & & \\
\hline
\end{tabular}

As herein discovered, the LTR enhanced HRES-1/Rab4 expression to a greater extent in subjects with rs451401/CC alleles relative to subjects with rs451401/GG alleles. CpG motifs flanking rs451401 were hypomethylated in SLE. CpG position 229407630 (Supplemental Figure 3) was earlier found less methylated in lupus B cells (38), also indicating an overall hypomethylation of the LTR in SLE. Importantly, the methylation of cytosine ${ }^{+954}$ immediately adjacent to rs451401 influenced LTR binding by IRF2 and PSIP1. IRF2 preferentially bound to rs451401/GG LTR over rs451401/CC LTR; however, methylation reversed this trend. IRF2 suppressed HRES-1/Rab4 expression in $\mathrm{T}$ cells of donors with rs451401/GG alleles while it had opposite effects in donors with rs451401/CC alleles. Alternatively, PSIP1 increased HRES-1/Rab4 expression in donors with rs451401/GG alleles. Thus, HRES-1/Rab4 expression is regulated by IRF2 and PSIP1 in manners dependent on the genotype and methylation state of the LTR enhancer (Figure 8).

HRES-1/Rab4 overexpression causes lysosomal degradation of CD4 and, thus, it protects from HIV-1 infection (23). As shown here, IRF2 promotes the expression of HRES-1/Rab4 in subjects with rs451401/CC LTR. Interestingly, IRF2 also binds the CXCR4 promoter (39). Thus, IRF2 may regulate susceptibility to infection by HIV-1 through both coreceptors. Unlike IRF2, PSIP1 facilitated HRES$1 /$ Rab4 expression in subjects carrying rs451401/GG alleles. In addition to serving as a transcriptional coactivator, PSIP1 binds HIV-1 integrase (40) that is being exploited to inhibit viral pathogenesis (41). Given that binding of each of these TFs to the HRES-1 LTR is methylation dependent, their interaction is highly likely to be influenced by rs451401 and epigenetic actors. Of note, PSIP1 interacts with ASF/SF2 to modulate pre-mRNA splicing $(42,43)$. As unveiled by this study, induction of PSIP1 by TCR stimulation occurred with a reduction in molecular weight of IRF1. This may involve alternative splicing of IRF-1 by ASF/SF2 (44), which is similarly overexpressed in lupus T cells $(45,46)$.

TCR activation induced the expression of NRF1, USF1, IRF2 and PSIP1, which was markedly enhanced in subjects with rs451401/CC alleles who evidently overexpress HRES-1/Rab4. Importantly, HRES-1/Rab4 overexpression in lupus T cells depends on mTOR activation (21). In turn, protein levels of mTORC1 substrates 4E-BP1 and S6K were elevated in subjects with rs451401/CC alleles. Given that TCR stimulation preferentially increased the expression of HRES-1/Rab4 as well as mTORC1 in subjects with rs451401 CC alleles, it plausible that more pronounced upregulation of USF1 and PSIP1 is mediated by greater mTORC1 activity via enhanced protein translation (47). Activation of mTORC1 and blockade of mTORC2 by HRES-1/Rab4 mimic the changes documented in lupus T cells (48) that can be attributed to inhibitory phosphorylation of mTORC2 by mTORC1 (49). Given that mTOR is located on HRES-1/ Rab4-positive endosomes (21), HRES-1/Rab4 may form a positive feedback loop with mTORC1 during TCR-initiated signal transduction. Importantly, mTORC1 blockade with rapamycin or Rab GTPase blockade with 3-PEHPC abrogated $\mathrm{T}$ cell activation, autoantibody production, and nephritis in lupus-prone mice (22). mTORC1 blockade with NAC (50) or rapamycin demonstrated therapeutic efficacy in SLE patients (51). As mounting evidence indicates, mTORC1 activation in tuberous sclerosis and lymphangioleiomyomatosis patients can lead to development of severe SLE (44, 52-56). Therefore, future studies are warranted to delineate the role of HRES-1/Rab4 polymorphism as a genetic cause of mTORC1 activation and predictor of clinical responsiveness to mTOR blockade in SLE and mechanistically connected disease conditions (35).

\section{Methods}

Human subjects. A total of 165 female White patients with SLE were investigated. All patients satisfied the criteria for a definitive diagnosis $(57,58)$. As controls, 111 White healthy control females were examined. The age of SLE patients was $42.2 \pm 1.1$ years (range 18-68 years), while that of healthy control subjects was $41.3 \pm 2.3$ years (range 18-63 years). 


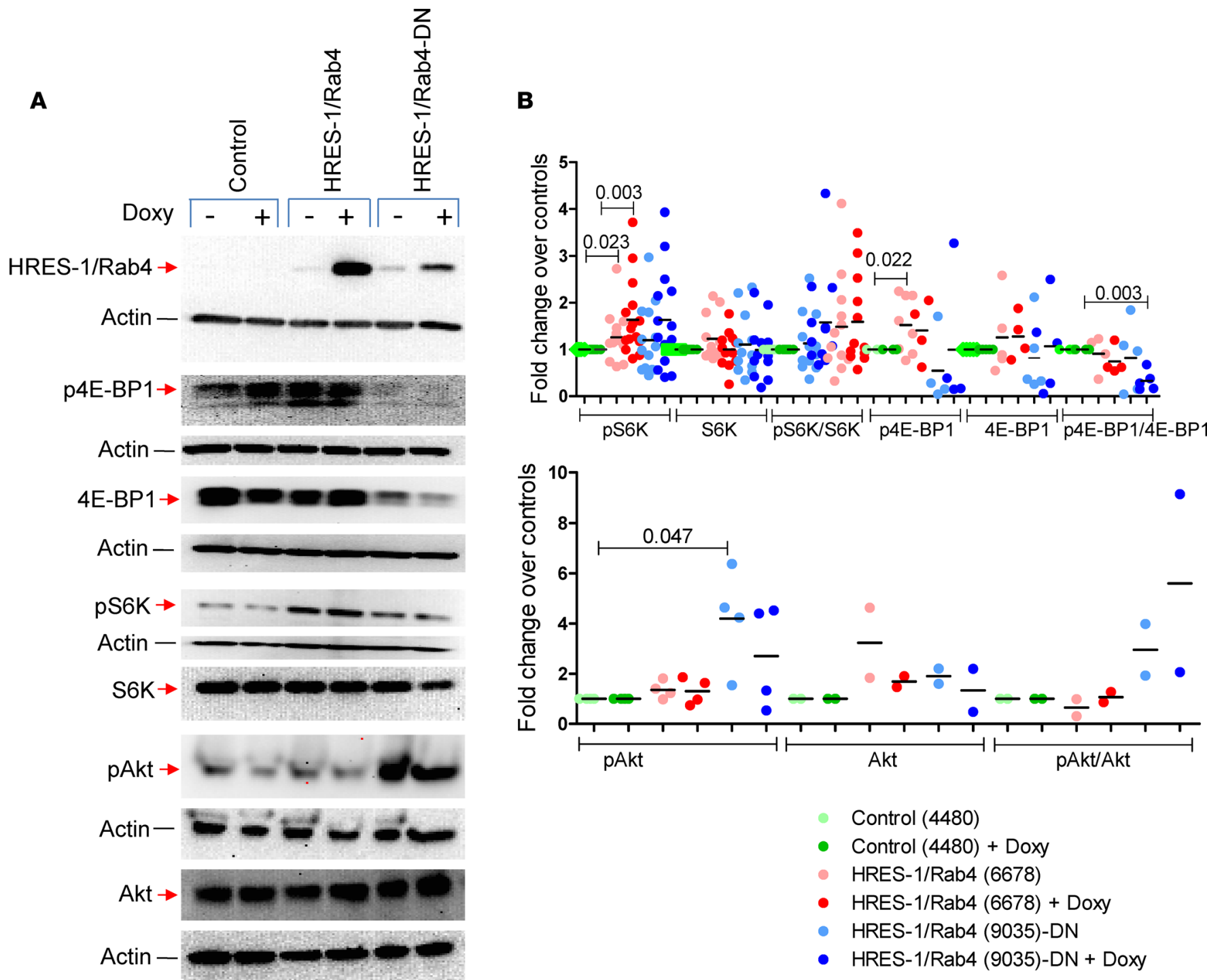

Figure 8. Western blot analysis of mTOR pathway activation by HRES-1/Rab4 in Jurkat cells. Activation of mTOR complexes 1 (mTORC1) and 2 (mTORC2) was assessed through phosphorylation of key substrates of mTORC1 (S6K, 4E-BP1) and mTORC2 (Akt). (A) Representative Western blots. (B) Cumulative analyses (mean \pm SEM). Means were normalized to control Jurkat cells carrying doxycycline-inducible expression vector (construct 4480) in the absence or presence of doxycycline. Jurkat cells with construct 6678 overexpressed wild-type HRES1-1/Rab4, while those with construct 9035 overexpressed dominant-negative HRES-1/Rab4527N. $P$ values represent comparison using 2 -tailed paired $t$ test, which reflect hypothesis testing and have not been corrected for multiple comparisons.

Separation and culture of human PBLs. Peripheral blood mononuclear cells were isolated from heparinized venous blood on a Ficoll-Hypaque gradient. PBLs were separated from monocytes by adherence to autologous serum-coated Petri dishes (59). T cells $\left(>95 \% \mathrm{CD}^{+}\right)$were negatively isolated from peripheral blood mononuclear cells and stimulated with CD3/CD28 (21), as described in the Supplemental Methods.

Cell lines. HeLa and HeLa-tat human cervix carcinoma cells (ATCC) were grown in Dulbecco's Modified Eagle's Medium with 10\% FBS, $2 \mathrm{mM}$ L-glutamine, $100 \mathrm{U} / \mathrm{ml}$ penicillin, $100 \mathrm{\mu g} / \mathrm{ml}$ streptomycin, and $10 \mu \mathrm{g} / \mathrm{ml}$ amphotericin B. HCT116 cells (ATCC) were cultured in McCoy's 5A modified medium supplemented with $10 \% \mathrm{FBS}$ and $1 \%$ penicillin/streptomycin/amphotericin B (60). HCT-166 colon carcinoma cells with wild-type DNMT1 and DNMT3 alleles (line no. 28) and HCT-166 colon carcinoma cells lacking DNMT1 (line no. 30), DNMT3b (line no. 38), or both DNMT1 and DNMT3b (DNMT1/3-DKO, line no. 343) were provided by Bert Vogelstein (Johns Hopkins University, Baltimore, Maryland, USA) (60). MCF7 cells expressing wild-type and dominant-negative IRF1 were obtained from Robert Clarke (Georgetown University, Washington, DC, USA) (61). Jurkat human T cell leukemia cells were maintained in RPMI 

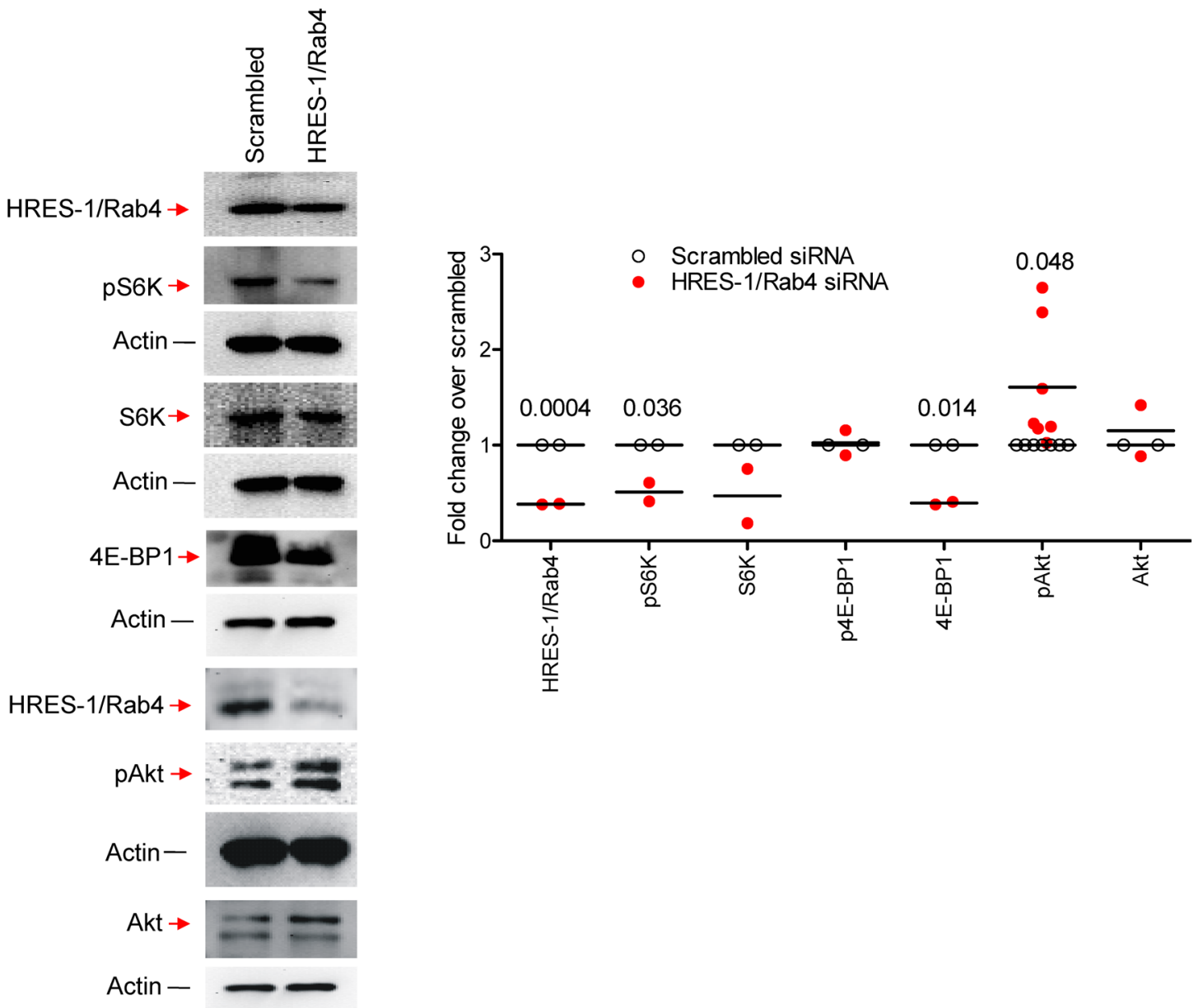

Figure 9. Western blot analysis of mTOR pathway activation of normal human T cells upon siRNA-mediated knockdown of HRES-1/Rab4.

Following siRNA transfection T cells were activated through CD3/CD28 for 24 hours. Data represent 2 or more independent experiments. $P$ values reflect comparison with paired $t$ test.

1640 medium supplemented with 10\% FBS, 2 mM L-glutamine, and antibiotics. All cell lines were maintained in a humidified atmosphere with $5 \% \mathrm{CO}_{2}$ at $37^{\circ} \mathrm{C}$, as earlier described (23). Control and HIV-1 tat gene-transfected HeLa and Jurkat cells were obtained from the NIH AIDS Research and Reference Program. Cell culture products were purchased from Cellgro (Mediatech Inc.).

Preparation of nuclear extracts. Nuclear extracts (NEs) were prepared as described in the Supplemental Methods.

EMSA. Single-stranded oligonucleotides were customer synthesized at Integrated DNA Technologies. Double-stranded synthetic oligonucleotides (Figure 4) were generated by heating primer pairs (see Figure $4 \mathrm{~A}$ and Supplemental Figures $6-8$ and 11 ) to $95^{\circ} \mathrm{C}$ for 5 minutes in $100 \mathrm{mM} \mathrm{NaCl}, 10 \mathrm{mM}$ Tris (pH 8), 1 $\mathrm{mM}$ EDTA and slow cooling to room temperature. They were aliquoted and stored at $-20^{\circ} \mathrm{C}$ until further use. These double-stranded oligonucleotides were 5'-end-labeled with 4,500 Ci/mmol $\left[\gamma_{-}{ }^{32} \mathrm{P}\right]$-ATP (Gamma-32, MP Biomedicals; catalog no. 0135020) and T4 polynucleotide kinase (Promega; catalog no. M4101) for 1 hour at $37^{\circ} \mathrm{C}$ and purified on Sephadex G-25 spin columns for use as EMSA probes (MilliporeSigma; GE Healthcare, catalog no. 28-9034-08). $10 \mu \mathrm{g}$ NE was incubated with $20 \mathrm{fmol}(45,000-60,000 \mathrm{cpm})$ of double-stranded probe in EMSA binding buffer $[20 \mathrm{mM}$ Tris $\mathrm{pH} 7.6,50 \mathrm{mM} \mathrm{NaCl}, 1 \mathrm{mM} \mathrm{MgCl}, 0.2 \mathrm{mM}$ EDTA, $5 \%$ glycerol, $25 \mathrm{ng} / \mu 1$ poly (dI-dC) poly(dI-dC) or $25 \mathrm{ng} / \mu 1$ sonicated salmon sperm DNA, $30 \mu \mathrm{g}$ of BSA, $0.1 \mathrm{mM}$ PMSF, and $0.5 \mathrm{mM} \mathrm{DTT}]$ for 30 minutes at room temperature in a total volume of $20 \mu 1$ in the presence or absence of 10- to 100-fold molar excess cold competitor DNA. In supershift experiments, 2 $\mu 1 \mathrm{TF}$-specific antibody or actin control antibody was preincubated with NE in EMSA binding buffer for 1 hour on ice followed by a 30-minute incubation with the ${ }^{32} \mathrm{P}$-labeled probe at room temperature. Antibodies used for supershift analyses and imaging are described in the Supplemental Methods. 


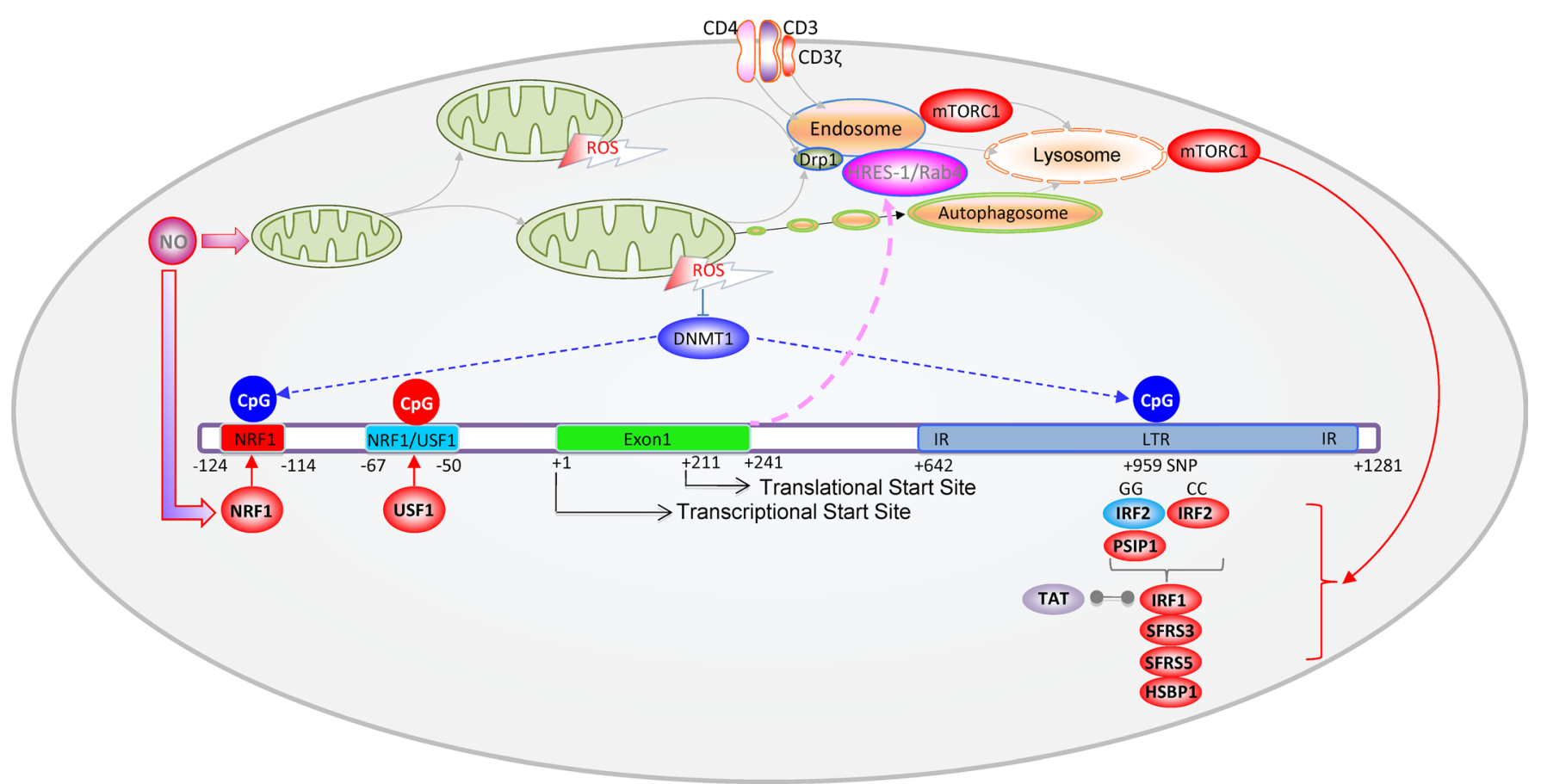

Figure 10. Schematic diagram of transcriptional regulation of HRES-1 expression and its effect on $\mathbf{T}$ cell activation. Promoter activity is controlled by binding of NRF1 and USF1 to nucleotides -50 to -67 (site 1) and -114 to -124 (site 2), respectively. NRF1 binds to site 1 when demethylated at cytosine-51 while it only binds site 2 when it is methylated at cytosine ${ }^{-119}$. Alternatively, USF1 only binds site 2 when cytosine $\mathrm{e}^{-19}$ is methylated. Expression of HRES-1/ Rab4 is enhanced by the LTR in intron 1. LTR enhancer activity is regulated by transcription factors IRF2, PSIP1, IRF, SFRS3, SFRS5, and HSBP1. As earlier documented, IRF1 interacts with HIV-tat (62). Binding of IRF2 and PSIP1 is influenced by the SNP rs451401 with CC alleles promoting IRF2, while GG alleles promote PSIP1 activity in a cell type-specific manner. HRES1/Rab4 expression is elevated in PBLs from human subjects with rs451401 CC alleles relative to those with GG alleles with and without TCR stimulation. Human subjects with rs451401 CC alleles show enhanced CD3/CD28 costimulation-induced activation of mTORC1 and transcription factors that regulate the expression of HRES-1/Rab4. Inactivation of DNMT1 promotes the expression of HRES-1/ Rab4. Methylation is reduced within the LTR enhancer and site 1 (cytosine ${ }^{-51}$ ) of the promoter, while it is increased in site 2 (cytosine ${ }^{-119}$ ) of the promoter in patients with SLE that all favor overexpression of HRES-1/Rab4. HRES-1/Rab4 mediates the endocytic recycling and lysosomal degradation of CD4, CD3 $\zeta$, and Drp1 $(21,22)$. The loss of Drp1 blocks mitophagy and promotes the accumulation of oxidative stress-generating mitochondria (22, 63). The resultant

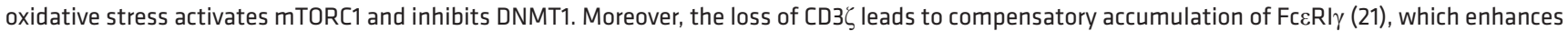
TCR-mediated signal transduction (64). Thus, human subjects with rs451401 CC alleles accommodate a positive feedback loop involving TCR signal transduction, mTOR activation, and DNMT1 inhibition, which synergistically promote the overexpression of HRES-1/Rab4.

Site-directed mutagenesis and deletion of reporter plasmids. Mutagenesis of HRES-1/Rab4 promoter and LTR enhancer constructs were generated by PCR using the QuikChange Site-Directed Mutagenesis Kit per the manufacturer's instructions (Stratagene). Additional details are provided in the Supplemental Methods.

Transient transfections and reporter gene assays. HeLa and HeLa-tat cells were transfected with pGL4.11 (Promega, catalog no. E6661) firefly luciferase plasmid vector-based reporter constructs using LipofectAMINE Plus reagent (Invitrogen). Each reporter plasmid was cotransfected with Renilla luciferase vector, hRluc/TK (pGL4.74, Promega, catalog no. E6921), to normalize for potential variation in transfection efficiency. Promoter DNA was cloned into Kpn1 and Xho1 sites of pGL4.11 vector. LTR enhancer was inserted into the BamH1 site of pGL4.11. The conversion of genotype I LTR to genotype II LTR was carried out using the Site Directed Mutagenesis kit (Stratagene/Agilent Technologies, catalog no. 200519-5). For each transfection, HeLa and HeLa-tat cells were plated at a cell density of $2.5 \times 10^{4}$ cells/well in a 48 -well plate 24 hours prior to transfection. Cells in each well were transfected with $200 \mathrm{ng}$ luciferase reporter vector and 50 ng Renilla control vector using Lipofectamine PLUS Reagent (Invitrogen, catalog no. 15338-100). Forty-eight hours after transfection, cells were harvested. Luciferase activity was assayed using the Dual-Glo System (Promega, catalog no. E2920). Luminescence signals for Firefly and Renilla luciferase were quantified with a BIOTEK bioluminescence plate reader. For all transfections, the relative light unit value of the Firefly luciferase signal was normalized to the value of Renilla luciferase signal to evaluate promoter activity.

Yeast 1-hybrid assay. The Matchmaker One Hybrid kit was used for construction and screening of the cDNA expression library (Clontech-Takara, catalog no. 630304, version PT3529-1). The LTR sequence between nucleotides +945 and +965 from the transcription start site of HRES-1/Rab4 was used as a DNA bait. 
Three copies of the DNA bait were inserted between the EcoR1 and Mlu1 restriction sites of the pHIS2.1 vector. Single-stranded oligonucleotides were custom synthesized by Integrated DNA Technologies and made into double-stranded DNA prior to cloning into pHIS2.1. For cDNA library construction, exponentially growing Jurkat E.6.1 cells were used for extracting total RNA and isolating mRNA with oligo-dT. The cDNA construction and PCR amplification of the CDNA library was carried out as suggested by the Matchmaker protocol. The mRNA was reverse transcribed using enzyme MMLV reverse transcriptase and oligonucleotide primers CDSIII Oligo dT [5'ATTCTAGAGGCCGACCGACATG-d(T) 30 VN-3'] and SMARTIII oligo [5'AAGCAGTGGTATCAACGCAGAGTGGCCATTATGGCCGGG-3']. The generated cDNA was amplified using 5' PCR primer 5'-TTCCACCCAAGCAGTGGTATCAACGCAGAGTGG-3' (homologous to SMART III oligo) and 3' PCR Primer 5'-GTATCGATGCCCACCCTCTAGAGGCCGAGGCGGCCGACA-3' (homologous to CDSIII oligo dT). Yeast cells were used for transformation with PCR-amplified cDNA library and Sma1-linearized pGADT7-Rec2 plasmid. The positive colonies were selected in SD/-Trp/-His/-Leu/+50 mM 3-AT media 3-5 days after transformation. Plasmids were isolated from the positive yeast colonies after overnight culture in SD/-Leu media. This plasmid DNA was sequenced with the $5^{\prime} \mathrm{T} 7$ primer annealing $5^{\prime}$ of the cDNA insert in the pGADT7-Rec2 cloning vector. Additional details are provided in the Supplemental Methods.

Trypsin digestion and mass spectrometry. Mass spectrometry was carried out on peptides generated through trypsin digestion of proteins that had exhibited binding to NRF1 sites 1 (nucleotides -50 to -67 ) and 2 (nucleotides -114 to -124 ) of the $5^{\prime}$ promoter and to the HRES1 LTR enhancer (nucleotides +945 to +965). Trypsin-digested samples were air-dried and resuspended in $25 \mu 1$ of $1 \%$ TFA solution and then were used for mass spectrometry with the Thermo LTQ Orbitrap instrument. Additional details are provided in the Supplemental Methods.

ChIP. ChIP assay was carried out using the Zymo-Spin ChIP Kit (Zymo Research) according to the manufacturer's instructions. Jurkat or HeLa cells were cross-linked for 7 minutes in PBS with 1\% (v/v) formaldehyde, after which they were quenched by incubation with $125 \mathrm{mM}$ glycine for 5 minutes. Cell extracts were incubated overnight with antibodies, and chromatin-protein complexes were eluted and reverse cross-linked through incubation with proteinase $\mathrm{K}$ for 90 minutes at $65^{\circ} \mathrm{C}$. After addition of ChIP DNA Binding Buffer (MilliporeSigma), the eluates were added to Zymo-Spin IC columns, and ChIP DNA was eluted in DNA Elution Buffer. ChIP efficacy was evaluated through end-point PCR using primers detecting NRF1 sites 1 and 2 and the LTR enhancer, as described in the Supplemental Methods.

Cloning of TF $c D N A$. Total RNA from exponentially growing Jurkat cells was isolated using the RNeasy Protected Mini kit (Qiagen, catalog no. 74124). From total RNA, mRNA was isolated using the Oligotex mRNA Mini kit (Qiagen, catalog no. 70022). 100 ng of mRNA was reverse transcribed using Superscript RT II (Invitrogen, catalog no. 11904-018). Individual TFs were amplified using gene-specific primers (see Supplemental Methods), which also contained the site for restriction enzyme digestion. The ORFs were cloned into the EcoRI and SalI sites of the multiple cloning site within pAAV-IRES-hrGFP plasmid. The ORF for IRF2 was purchased from Origene (catalog no. SC118745, pCMVXL5 containing IRF2 cDNA). After ligation, plasmids were transfected into E. coli cells (Stratagene, XL-Gold, catalog no. 200314), and transformants were selected in media containing $50 \mu \mathrm{g} / \mathrm{ml}$ ampicillin. HEK-293-derived AAV-293 cells were transfected with plasmids pRC, pHelper, and pAAV-IRES-hrGFP, containing TF ORFs to produce AAV.

Construction and production of $A A V$. TFs were transduced into PBLs by infection with AAV carrying cDNA upstream of the internal ribosomal entry site (IRES) within the pAAV-IRES-hrGFP vector (Stratagene/Agilent Technologies, catalog no. 240071), as described previously (23). Transduction was verified by flow cytometry of $10^{5}$ cells per sample (LSRII, BD Biosciences). The remainder of the cells were lysed directly at a density of $2 \times$ $10^{6}$ live cells per $100 \mu 1$ of $4 \times$ SDS-PAGE sample buffer. GFP expression was monitored by flow cytometry and equilibrated among constructs (excitation: 488 nm, emission: 509 nm; FL1 channel). Optimal GFP expression occurred at 48 hours after infection of PBLs with $>99 \%$ of cells infected. Primary PBLs and T cells were analyzed 24 hours after infection, due to a decline in cell viability at 48 hours. Overexpression of TFs was confirmed by Western blotting. Additional details are provided in the Supplemental Methods.

Transfection of HeLa and HeLa-tat cells with TFs. HeLa and HeLa-tat cells were transfected with pAAV-IRES-hrGFP containing TF ORFs. The cells were plated at a cell density of $1 \times 10^{5}$ cells/well in a 6-well plate 24 hours prior to transfection. The cells were transfected with Lipofectamine PLUS Reagent. Twenty-four hours and forty-eight hours after transfection, the cells were harvested through trypsinization, and an aliquot was used to detect GFP signal through flow cytometry to confirm transfection. Protein lysates were prepared as described for PBLs. 
Western blot analyses. Whole-cell protein lysates were prepared by lysis in radioimmunoprecipitation assay buffer (150 mM NaCl, 2\% NP-40, 0.5\% sodium deoxycholate, 0.1\% SDS, $50 \mathrm{mM}$ Tris pH 8.0, $1 \mathrm{mM}$ PMSF, $1 \mu \mathrm{g} / \mathrm{ml}$ aprotinin, $1 \mu \mathrm{g} / \mathrm{ml}$ pepstatin, $1 \mu \mathrm{g} / \mathrm{ml}$ leupeptin, $1 \mathrm{mM} \mathrm{NaF}, 1 \mathrm{mM}$ sodium orthovanadate, $0.1 \mathrm{mM}$ sodium molybdate, $10 \mathrm{mM}$ sodium pyrophosphate) at a density of $4 \times 10^{7} \mathrm{cells} / \mathrm{ml}$ on ice, followed by addition of equal volumes of Laemmli protein sample buffer ( $60 \mathrm{mM}$ Tris-Cl pH 6.8, 2\% SDS, $10 \%$ glycerol, $5 \% \beta$-mercaptoethanol, $0.01 \%$ bromophenol blue), and heated to $95^{\circ} \mathrm{C}$ for 5 minutes prior to separation on SDS-PAGE gels and transfer to $0.45-\mu \mathrm{m}$ nitrocellulose membranes. As loading control, actin antibody was placed onto each membrane to accurately authenticate changes in expression of each specific gene. Antibodies and imaging are described in the Supplemental Methods.

Statistics. Statistical analyses were performed with Prism version 5.0 for Windows (GraphPad Software). Data were expressed as the mean \pm SEM of individual experiments. Data are presented as dot plots or box-and-whiskers plots. For hypothesis testing, changes were considered significant at $P<0.05$. Statistical analysis was corrected for multiple comparisons when more than 2 groups were assessed in parallel, as stated in the Results section and each figure legend, as appropriate.

Study approval. Involvement of human subjects in the study has been approved by the Institutional Review Board and the Institutional Biosafety Committee of the State University of New York Upstate Medical University.

\section{Author contributions}

AG, RK, JJ, MB, TC, DF, NH, MD, JL, HJF, EMP, KB, and AP meaningfully contributed to the design, execution, and interpretation of the study.

\section{Acknowledgments}

This work has been supported in part by grants AI 048079, AI 072648, and AI 122176 from the NIH and the Central New York Community Foundation. The authors thank Robert Clarke (Georgetown University) and Bert Vogelstein (Johns Hopkins University) for sharing reagents.

Address correspondence to: Andras Perl, State University of New York, College of Medicine, 750 East Adams Street, Syracuse, New York 13210. Phone: 315.464.4194; Email: perla@upstate.edu.

1. Perl A. Systems biology of lupus: mapping the impact of genomic and environmental factors on gene expression signatures, cellular signaling, metabolic pathways, hormonal and cytokine imbalance, and selecting targets for treatment. Autoimmunity. 2010;43(1):32-47.

2. Li Y, Gorelik G, Strickland FM, Richardson BC. Oxidative stress, T cell DNA methylation and lupus. Arthritis Rheum. 2014;66(6):1574-1582.

3. Weeding E, Sawalha AH. Deoxyribonucleic acid methylation in systemic lupus erythematosus: implications for future clinical practice. Front Immunol. 2018;9:875.

4. Absher DM, et al. Genome-wide DNA methylation analysis of systemic lupus erythematosus reveals persistent hypomethylation of interferon genes and compositional changes to CD4+ T-cell populations. PLoS Genet. 2013;9(8):e1003678.

5. Coit $\mathrm{P}$, et al. Epigenome profiling reveals significant DNA demethylation of interferon signature genes in lupus neutrophils J Autoimmun. 2015;58:59-66.

6. Crow MK, Kirou KA. Interferon-alpha in systemic lupus erythematosus. Curr Opin Rheumatol. 2004;16(5):541-547.

7. Denny MF, et al. Interferon-alpha promotes abnormal vasculogenesis in lupus: a potential pathway for premature atherosclerosis. Blood. 2007;110(8):2907-2915.

8. Chiappinelli KB, et al. Inhibiting DNA methylation causes an interferon response in cancer via dsRNA including endogenous retroviruses. Cell. 2015;162(5):974-986.

9. Boeke JD, Stoye JP. Retrotransposons, endogenous retroviruses, and the evolution of retroelements. In Coffin JM, Hughes SH, Varmus HE, eds. Retroviruses. Cold Spring Harbor, New York, USA: Cold Spring Harbor Laboratory Press;2011:343-435.

10. Perl A, Banki K. Human endogenous retroviral elements and autoimmunity: data and concepts. Trends Microbiol. 1993;1(4):153-156.

11. Perl A, Fernandez D, Telarico T, Phillips PE. Endogenous retroviral pathogenesis in lupus. Curr Opin Rheumatol. 2010;22(5):483-492.

12. Tsao BP. Lupus susceptibility genes on human chromosome 1. Int Rev Immunol. 2000;19(4-5):319-334.

13. Magistrelli C, et al. Polymorphic genotypes of the HRES-1 human endogenous retrovirus locus correlate with systemic lupus erythematosus and autoreactivity. Immunogenetics. 1999;49(10):829-834.

14. Pullmann R, Bonilla E, Phillips PE, Middleton FA, Perl A. Haplotypes of the HRES-1 endogenous retrovirus are associated with development and disease manifestations of systemic lupus erythematosus. Arthritis Rheum. 2008;58(2):532-540.

15. Rasmussen HB, Christensen K, Clausen J. Sequencing and detection of polymorphisms in the 5' end of the human endogenous retroviral element, HRES-1. Dis Markers. 1996;13(1):1-9.

16. Rasmussen HB, Kelly MA, Francis DA, Clausen J. Association between the endogenous retrovirus HRES-1 and multiple sclero- 
sis in the United Kingdom--evidence of genetically different disease subsets? Dis Markers. 2000;16(3-4):101-104.

17. Rasmussen HB, Clausen J. A novel haplotype of the endogenous retrovirus, HRES-1, in patients with multiple sclerosis and healthy individuals. Autoimmunity. 1999;29(2):141-145.

18. Rasmussen HB, Kelly MA, Francis DA, Clausen J. Haplotypes of the endogenous retrovirus HRES-1 in multiple sclerosis patients and healthy control subjects of Shanghai Chinese origin. Dis Markers. 1998;13(4):251-255.

19. Rasmussen HB, Heltberg A, Christensen K, Clausen J. Possible association between multiple sclerosis and the human T cell leukemia virus (HTLV)-related endogenous element, HRES-1. Mult Scler. 1996;2(3):133-136.

20. Rasmussen HB, et al. Expression of endogenous retroviruses in blood mononuclear cells and brain tissue from multiple sclerosis patients. Mult Scler. 1995;1(2):82-87.

21. Fernandez DR, et al. Activation of mammalian target of rapamycin controls the loss of TCRzeta in lupus T cells through HRES-1/Rab4-regulated lysosomal degradation. J Immunol. 2009;182(4):2063-2073.

22. Caza TN, et al. HRES-1/Rab4-mediated depletion of Drp1 impairs mitochondrial homeostasis and represents a target for treatment in SLE. Ann Rheum Dis. 2014;73(10):1888-1897.

23. Nagy G, et al. Regulation of CD4 expression via recycling by HRES-1/RAB4 controls susceptibility to HIV infection. $J$ Biol Chem. 2006;281(45):34574-34591.

24. Quandt K, Frech K, Karas H, Wingender E, Werner T. MatInd and MatInspector: new fast and versatile tools for detection of consensus matches in nucleotide sequence data. Nucleic Acids Res. 1995;23(23):4878-4884.

25. Cartharius K, et al. MatInspector and beyond: promoter analysis based on transcription factor binding sites. Bioinformatics. 2005;21(13):2933-2942.

26. Wang J, et al. Sequence features and chromatin structure around the genomic regions bound by 119 human transcription factors. Genome Res. 2012;22(9):1798-1812.

27. Perl A, et al. Detection and cloning of new HTLV-related endogenous sequences in man. Nucleic Acids Res. 1989;17(17):6841-6854

28. Yung R, Chang S, Hemati N, Johnson K, Richardson B. Mechanisms of drug-induced lupus. IV. Comparison of procainamide and hydralazine with analogs in vitro and in vivo. Arthritis Rheum. 1997;40(8):1436-1443.

29. Scarpulla RC. Transcriptional paradigms in mammalian mitochondrial biogenesis and function. Physiol Rev. 2008;88(2):611-638.

30. Evans MJ, Scarpulla RC. Interaction of nuclear factors with multiple sites in the somatic cytochrome c promoter. Characterization of upstream NRF-1, ATF, and intron Sp1 recognition sequences. J Biol Chem. 1989;264(24):14361-14368.

31. Calomme $\mathrm{C}$, et al. Upstream stimulatory factors binding to an $\mathrm{E}$ box motif in the $\mathrm{R}$ region of the bovine leukemia virus long terminal repeat stimulates viral gene expression. J Biol Chem. 2002;277(11):8775-8789.

32. Merry TL, Ristow M. Nuclear factor erythroid-derived 2-like 2 (NFE2L2, Nrf2) mediates exercise-induced mitochondrial biogenesis and the anti-oxidant response in mice. J Physiol (Lond). 2016;594(18):5195-5207.

33. Grossman CE, Qian Y, Banki K, Perl A. ZNF143 mediates basal and tissue-specific expression of human transaldolase. J Biol Chem. 2004;279(13):12190-12205.

34. Sgarbanti M, et al. IאB kinase $\varepsilon$ targets interferon regulatory factor 1 in activated T lymphocytes. $\mathrm{Mol}$ Cell Biol. 2014;34(6):1054-1065

35. Perl A. mTOR activation is a biomarker and a central pathway to autoimmune disorders, cancer, obesity, and aging. Ann $N Y$ Acad Sci. 2015;1346(1):33-44.

36. Vallet VS, et al. Differential roles of upstream stimulatory factors 1 and 2 in the transcriptional response of liver genes to glucose. J Biol Chem. 1998;273(32):20175-20179.

37. Li H, et al. Precision DNA demethylation ameliorates disease in lupus-prone mice. JCI Insight. 2018;3(16):120880.

38. Fali T, et al. DNA methylation modulates HRES1/p28 expression in B cells from patients with Lupus. Autoimmunity. 2014;47(4):265-271.

39. Finley MJ, Steele A, Cornwell WD, Rogers TJ. Transcriptional regulation of the major HIV-1 coreceptor, CXCR4, by the kappa opioid receptor. J Leukoc Biol. 2011;90(1):111-121.

40. Llano M, et al. An essential role for LEDGF/p75 in HIV integration. Science. 2006;314(5798):461-464.

41. Christ F, et al. Rational design of small-molecule inhibitors of the LEDGF/p75-integrase interaction and HIV replication. Nat Chem Biol. 2010;6(6):442-448.

42. Ge H, Si Y, Wolffe AP. A novel transcriptional coactivator, p52, functionally interacts with the essential splicing factor ASF/ SF2. Mol Cell. 1998;2(6):751-759.

43. Hanamura A, Cáceres JF, Mayeda A, Franza BR, Krainer AR. Regulated tissue-specific expression of antagonistic pre-mRNA splicing factors. $R N A$. 1998;4(4):430-444.

44. Tzoanopoulos D, et al. Low expression of interferon regulatory factor-1 and identification of novel exons skipping in patients with chronic myeloid leukaemia. Br J Haematol. 2002;119(1):46-53.

45. Lemaire R, Winne A, Sarkissian M, Lafyatis R. SF2 and SRp55 regulation of CD45 exon 4 skipping during T cell activation. Eur J Immunol. 1999;29(3):823-837.

46. Moulton VR, Grammatikos AP, Fitzgerald LM, Tsokos GC. Splicing factor SF2/ASF rescues IL-2 production in T cells from systemic lupus erythematosus patients by activating IL-2 transcription. Proc Natl Acad Sci USA. 2013;110(5):1845-1850.

47. Sonenberg N, Hinnebusch AG. Regulation of translation initiation in eukaryotes: mechanisms and biological targets. Cell. 2009;136(4):731-745.

48. Kato H, Perl A. Mechanistic target of rapamycin complex 1 expands Th17 and IL-4+ CD4-CD8-double-negative T cells and contracts regulatory T cells in systemic lupus erythematosus. J Immunol. 2014;192(9):4134-4144.

49. Yang Q, Inoki K, Kim E, Guan KL. TSC1/TSC2 and Rheb have different effects on TORC1 and TORC2 activity. Proc Natl Acad Sci USA. 2006;103(18):6811-6816.

50. Lai ZW, et al. N-acetylcysteine reduces disease activity by blocking mammalian target of rapamycin in T cells from systemic lupus erythematosus patients: a randomized, double-blind, placebo-controlled trial. Arthritis Rheum. 2012;64(9):2937-2946.

51. Lai ZW, et al. Sirolimus in patients with clinically active systemic lupus erythematosus resistant to, or intolerant of, conventional medications: a single-arm, open-label, phase 1/2 trial. Lancet. 2018;391(10126):1186-1196.

52. Singh N, Birkenbach M, Caza T, Perl A, Cohen PL. Tuberous sclerosis and fulminant lupus in a young woman. J Clin Rheumatol. 2013;19(3):134-137. 
53. Olde Bekkink M, Ahmed-Ousenkova YM, Netea MG, van der Velden WJ, Berden JH. Coexistence of systemic lupus erythematosus, tuberous sclerosis and aggressive natural killer-cell leukaemia: coincidence or correlated? Lupus. 2016;25(7):766-771.

54. Carrasco Cubero C, Bejarano Moguel V, Fernández Gil MÁ, Álvarez Vega JL. Coincidence of tuberous sclerosis and systemic lupus erythematosus-a case report. Reumatol Clin. 2016;12(4):219-222.

55. Suzuki K, et al. A case of lymphangioleiomyomatosis associated with endometrial cancer and severe systemic lupus erythematosus. BMC Cancer. 2016;16:390.

56. Psarelis S, Nikiphorou E. Coexistence of SLE, tuberous sclerosis and aggressive natural killer-cell leukaemia: Coincidence or correlated? Lupus. 2017;26(1):107-108

57. Tan EM, et al. The 1982 revised criteria for the classification of systemic lupus erythematosus. Arthritis Rheum. 1982;25(11):1271-1277.

58. Hochberg MC. Updating the American College of Rheumatology revised criteria for the classification of systemic lupus erythematosus. Arthritis Rheum. 1997;40(9):1725.

59. Perl A, Gonzalez-Cabello R, Láng I, Gergely P. Effector activity of OKT4+ and OKT8+ T-cell subsets in lectin-dependent cell-mediated cytotoxicity against adherent HEp-2 cells. Cell Immunol. 1984;84(1):185-193.

60. Rhee I, et al. DNMT1 and DNMT3b cooperate to silence genes in human cancer cells. Nature. 2002;416(6880):552-556

61. Bouker KB, et al. Interferon regulatory factor-1 mediates the proapoptotic but not cell cycle arrest effects of the steroidal antiestrogen ICI 182,780 (faslodex, fulvestrant). Cancer Res. 2004;64(11):4030-4039.

62. Sgarbanti M, et al. Modulation of human immunodeficiency virus 1 replication by interferon regulatory factors. $J$ Exp Med. 2002;195(10):1359-1370.

63. Talaber G, et al. HRES-1/Rab4 promotes the formation of LC3(+) autophagosomes and the accumulation of mitochondria during autophagy. PLoS ONE. 2014;9(1):e84392.

64. Moulton VR, Tsokos GC. T cell signaling abnormalities contribute to aberrant immune cell function and autoimmunity. J Clin Invest. 2015;125(6):2220-2227. 